MATHEMATICS OF COMPUTATION

Volume 70, Number 236, Pages 1353-1381

S 0025-5718(00)01264-3

Article electronically published on October 27, 2000

\title{
A POSTERIORI ERROR CONTROL IN LOW-ORDER FINITE ELEMENT DISCRETISATIONS OF INCOMPRESSIBLE STATIONARY FLOW PROBLEMS
}

\author{
CARSTEN CARSTENSEN AND STEFAN A. FUNKEN
}

\begin{abstract}
Computable a posteriori error bounds and related adaptive meshrefining algorithms are provided for the numerical treatment of monotone stationary flow problems with a quite general class of conforming and nonconforming finite element methods. A refined residual-based error estimate generalises the works of Verfürth; Dari, Duran and Padra; Bao and Barrett. As a consequence, reliable and efficient averaging estimates can be established on unstructured grids. The symmetric formulation of the incompressible flow problem models certain nonNewtonian flow problems and the Stokes problem with mixed boundary conditions. A Helmholtz decomposition avoids any regularity or saturation assumption in the mathematical error analysis. Numerical experiments for the partly nonconforming method analysed by Kouhia and Stenberg indicate efficiency of related adaptive mesh-refining algorithms.
\end{abstract}

\section{INTRODUCTION}

Adaptive finite element methods play an important practical role in computational fluid dynamics. They are often justified by a posteriori error estimates which provide computable upper and lower error bounds which then serve as error indicators. In this paper, we unify and refine the derivation of such residual error estimates for possibly nonlinear flow problems, such as the Stokes problem V2, V3, DDP] and certain monotone nonNewtonian flow problems $[\mathrm{BB}] \mathrm{P}$. The refinement enables a justification of averaging techniques which are quite popular in engineering applications.

In the presentation emphasis is on a unifying proof for conforming, nonconforming, and even a conforming-nonconforming scheme $[\mathrm{KS}$. Because of possible Neumann boundary conditions, we study the symmetric formulation which appears to be less frequently analysed in the mathematical literature. For notational simplicity we only give details for $2 \mathrm{D}$ regular triangulations but allow mixed inhomogeneous boundary data.

Given a Lipschitz continuous monotone mapping $\mathcal{A}: \mathbb{R}_{\text {sym }}^{2 \times 2} \rightarrow \mathbb{R}_{\text {sym }}^{2 \times 2}$, Dirichlet data $u_{D} \in H^{1}(\Omega)^{2}$ and right-hand sides $f \in L^{2}(\Omega)^{2}$ and $g \in L^{2}\left(\Gamma_{N}\right)^{2}$ in a bounded

Received by the editor July 24, 1997 and, in revised form, June 2, 1999 and January 6, 2000. 2000 Mathematics Subject Classification. Primary 65N30, 76D07.

Key words and phrases. NonNewtonian flow, Stokes problem, Crouzeix-Raviart element, nonconforming finite element method, a posteriori error estimates, adaptive algorithm, reliability, efficiency. 
Lipschitz domain $\Omega \subset \mathbb{R}^{2}$, find $u \in H^{1}(\Omega)^{2}$ and $p \in L^{2}(\Omega)$ which satisfy

$$
\begin{gathered}
\operatorname{div} \sigma+f=0 \quad \text { and } \quad \operatorname{div} u=0 \quad \text { in } \Omega, \\
\sigma=\mathcal{A}(\varepsilon(u))-p I \quad \text { and } \quad \varepsilon(u):=\left(\nabla u+\nabla u^{\top}\right) / 2 \quad \text { in } \Omega, \\
u=u_{D} \quad \text { on } \Gamma_{D} \quad \text { and } \sigma \cdot n=g \quad \text { on } \Gamma_{N} .
\end{gathered}
$$

The stress-strain relation $\sigma=\mathcal{A}(\varepsilon(u))-p I$ (where $I$ denotes the $2 \times 2$-unit matrix) models Newtonian fluids for a linear function $\mathcal{A}(\alpha)=2 \mu \alpha$ with viscosity $\mu>0 ; \varepsilon(u)$ is the linear Green strain rate for the velocity field $u$. Then (1.1)-(1.3) is the (stationary) Stokes problem in the symmetric form with mixed boundary conditions. Non-Newtonian flows, such as the Carreau law, are included as long as there are positive constants $c_{1}$ and $c_{2}$ such that the Lipschitz continuous function $\mathcal{A}: \mathbb{R}_{\text {sym }}^{2 \times 2} \rightarrow \mathbb{R}_{\text {sym }}^{2 \times 2}$ (where $\mathbb{R}_{\text {sym }}^{2 \times 2}$ denotes the set of real symmetric $2 \times 2$ matrices) satisfies, for all $\alpha, \beta \in \mathbb{R}_{\text {sym }}^{2 \times 2}$,

$$
\begin{gathered}
c_{1}|\alpha-\beta|^{2} \leq(\mathcal{A}(\alpha)-\mathcal{A}(\beta)):(\alpha-\beta), \\
|\mathcal{A}(\alpha)-\mathcal{A}(\beta)| \leq c_{2}|\alpha-\beta| .
\end{gathered}
$$

(Colon denotes the scalar product in $\mathbb{R}_{\text {sym }}^{2 \times 2}$, i.e., $\alpha: \beta=\sum_{j, k=1}^{2} \alpha_{j k} \beta_{j k}$.) The boundary $\Gamma:=\partial \Omega$ of a bounded Lipschitz domain $\Omega$ in $\mathbb{R}^{2}$ is split into a closed Dirichlet boundary $\Gamma_{D} \subseteq \Gamma$ with positive surface measure and the remaining Neumann boundary $\Gamma_{N}:=\Gamma \backslash \Gamma_{D}$. We mention that in the case $\Gamma_{N}=\emptyset$, the pressure $p$ is defined only up to a constant and we require $\int_{\Gamma} u_{D} \cdot n d s=0$.

The discrete problem is characterised by a (possibly nonconforming) discrete space $\mathcal{V} \times \mathcal{Q} \subset L^{2}(\Omega)^{2} \times L^{2}(\Omega)$ with respect to an underlying regular triangulation $\mathcal{T}$ of the domain $\Omega$. A discrete solution $\left(u_{h}, p_{h}\right)$ in (a subspace of) $L^{2}(\Omega)^{2} \times L^{2}(\Omega)$ is supposed to satisfy

$$
\begin{gathered}
\int_{\Omega} \mathcal{A}\left(\varepsilon_{\mathcal{T}}\left(u_{h}\right)\right): \varepsilon_{\mathcal{T}}\left(v_{h}\right) d x-\int_{\Omega} p_{h} \operatorname{div}_{\mathcal{T}} v_{h} d x \\
=\int_{\Omega} f \cdot v_{h} d x+\int_{\Gamma_{N}} g \cdot v_{h} d s \quad\left(v_{h} \in \mathcal{V}\right), \\
-\int_{\Omega} q_{h} \operatorname{div}_{\mathcal{T}} u_{h} d x=0 \quad\left(q_{h} \in \mathcal{Q}\right) .
\end{gathered}
$$

Since discrete functions may be discontinuous, a lower index $\mathcal{T}$ on differential operators (e.g., $\nabla_{\mathcal{T}} u_{h}, \operatorname{div}_{\mathcal{T}} v_{h}$, etc.) denotes their $\mathcal{T}$-elementwise action which may be different from their distributional meaning.

Remarks 1.1. (i) The continuity condition $\operatorname{div} u=0$ is usually utilised in the Stokes problem to replace the term $\operatorname{div} 2 \mu \varepsilon(u)$ in (1.1) by $\mu \Delta u$. In the resulting nonsymmetric formulation the natural Neumann boundary condition reads $\partial u / \partial n+p n=g$ and is correct from a variational point of view, but not from a physical perspective. Hence, if Neumann data arise in the problem, the symmetric formulation (1.1)(1.3) is the reasonable mathematical model. However, the analysis presented below applies to the omitted nonsymmetric formulation as well.

(ii) Stability results and a priori error estimates for mixed and nonconforming finite element spaces $\mathcal{V} \times \mathcal{Q}$ can be found in $[\overline{\mathrm{BF}}, \overline{\mathrm{BS}}, \overline{\mathrm{GR}}, \overline{\mathrm{KS}}$. It turns out that, in contrast to the nonsymmetric formulation, the nonconforming Crouzeix-Raviart elements are not uniformly stable for the Stokes problem [FM]. Instead, a nonconforming finite element method is stable where one component of the displacement 
is discretised with conforming linear elements and the other with nonconforming linear elements [KS, i.e., the trial space for the displacement field is $\mathcal{V}=\mathcal{V}_{1} \times \mathcal{V}_{2}$ where

$$
\mathcal{V}_{1}:=\left\{V \in C(\Omega): V \text { is affine on each } T \in \mathcal{T} \text { and vanishes on } \Gamma_{D}\right\},
$$

$$
\mathcal{V}_{2}:=\{V: V \text { is affine on each } T \in \mathcal{T} \text {, continuous at midpoints of inner }
$$$$
\text { element boundaries, and vanishes at midpoints of edges } \left.E \subset \Gamma_{D}\right\}
$$

(with usual modifications on $\Gamma_{D}$ for inhomogeneous boundary conditions for the trial space) and $\mathcal{Q}$ are the $\mathcal{T}$-piecewise constants (with vanishing integral mean over $\Omega$ if $\left.\Gamma_{N}=\emptyset\right)$.

(iii) A posteriori error estimates and adaptive mesh-refining algorithms are included in [DDP, P, V1, V2, V3, for the nonsymmetric formulation without Neumann boundary data.

(iv) The unique existence of exact solutions $(u, p)$ to (1.1)-1.3 and discrete solutions $\left(u_{h}, p_{h}\right)$ to (1.6)-(1.7) is discussed in the literature (see, e.g., $\mathrm{BB}, \mathrm{BF}$, Ci $, \mathrm{GR}, \mathrm{KS}, \mathrm{QV}, \mathrm{T}]$ and the references quoted therein). In this paper we therefore adopt the point of view that the continuous problem has a unique solution and there are (not necessarily unique) known functions $\left(u_{h}, p_{h}\right)$ with certain $\mathcal{T}$-elementwise regularity properties given to us which satisfy the Galerkin conditions (1.6)-(1.7). There is no stability assumption on the discrete problem and indeed, in this way, unstable methods are analysed as well in their a posteriori error control (but this is not to recommend generally the application of unstable schemes).

(v) The class of finite element spaces under consideration in this paper is characterised by the fact that the integral mean of the jump $\left[u_{h}\right]$ vanishes (or is at least small) across interior edges. We stress that we do not need any a priori, saturation, or stability assumption on the discretisation or regularity of the exact solution.

(vi) The refined error estimate of this paper was (for the Stokes problem) announced in $\mathrm{CV}$. The presented analysis results from a long term research, independent from [DDP], $[\mathrm{P}]$, and $[\mathrm{BB}]$, that started with mixed methods in [Ca1] and with the Stokes problem in [CJ].

In this paper, we establish a new residual-based efficient and (to some extent) reliable error estimate that applies to a general class of finite element discretisations. To describe the results in a simplified setting, suppose for (1.8)-(1.9), in this introduction that $f \in H^{1}(\Omega)$ and the (possibly discontinuous) discrete solution $\left(u_{h}, p_{h}\right)$ is $\mathcal{T}$-piecewise smooth, satisfies $\operatorname{div}_{\mathcal{T}} u_{h}=0, \int_{E \backslash \Gamma}\left[u_{h}\right] d s=0$, and $\int_{E \cap \Gamma_{D}}\left(u_{h}-u_{D}\right) d s=0$ for all $E \in \mathcal{E}$, where $\mathcal{E}$ denotes the set of all edges in $\mathcal{T}$. Suppose $\sigma_{h}:=\mathcal{A}\left(\varepsilon_{\mathcal{T}}\left(u_{h}\right)\right)-p_{h} I$ is a $\mathcal{T}$-piecewise polynomial of degree at most $k$.

For each $T \in \mathcal{T}$, define the element contribution to the residual-error bound $\eta_{R}^{2}:=\sum_{T \in \mathcal{T}} \eta_{R, T}^{2}$ by

$$
\eta_{R, T}^{2}:=h_{T}^{4}\|\nabla f\|_{L^{2}(T)}^{2}+\sum_{E \in \mathcal{E} \wedge E \subset \partial T} h_{E}\left(\left\|\left[\sigma_{h} n_{E}\right]\right\|_{L^{2}(E)}^{2}+\left\|\left[\partial u_{h} / \partial s\right]\right\|_{L^{2}(E)}^{2}\right) .
$$

Here $h_{T}$ and $h_{E}$ are diameters of an element $T \in \mathcal{T}$ and an edge $E \in \mathcal{E}$, respectively. The jump of a (possibly discontinuous) function $G$ across the inner edge $E$ is written $[G]$ with modifications according to boundary data, and $\partial / \partial s$ is the derivative along 
edges with respect to the arc-length (see Section 2 for details). All the contributions in (1.10) are computable residuals of (1.1)-(1.3) weighted with mesh-sizes.

Our first result shows that $\eta_{R}$ is a reliable a posteriori error estimate in the sense that there exists an $h$-independent positive constant $c_{3}$ such that

$$
\left\|\varepsilon_{\mathcal{T}}\left(u-u_{h}\right)\right\|_{L^{2}(\Omega)}^{2}+\left\|p-p_{h}\right\|_{L^{2}(\Omega)}^{2} \leq c_{3} \sum_{T \in \mathcal{T}} \eta_{R, T}^{2}=c_{3} \eta_{R}^{2} .
$$

Secondly, the estimate (1.11) is efficient in the sense that the reverse inequality holds with an $h$-independent positive constant $c_{4}$

$$
\eta_{R} \leq c_{4}\left(\left\|\varepsilon_{\mathcal{T}}\left(u-u_{h}\right)\right\|_{L^{2}(\Omega)}+\left\|p-p_{h}\right\|_{L^{2}(\Omega)}\right)+\text { h.o.t. }
$$

up to higher order terms h.o.t. which are known and generically of higher order.

In the error estimator (1.11), the edge contributions dominate. This gives rise to a ZZ-type averaging estimator for the stress field as in $\mathrm{CB}$. We prove for the conforming-nonconforming scheme (1.8)-(1.9) that

$$
\left\|\varepsilon_{\mathcal{T}}\left(u-u_{h}\right)\right\|_{L^{2}(\Omega)}^{2}+\left\|p-p_{h}\right\|_{L^{2}(\Omega)}^{2} \leq c_{5}\left\|\sigma_{h}-\sigma_{h}^{*}\right\|_{L^{2}(\Omega)}+\text { h.o.t. }
$$

even in a more local form. Here, h.o.t. are known terms being generically of higher order and $\sigma_{h}^{*}$ is a continuous (not necessarily symmetric) $\mathcal{T}$-piecewise affine approximation to the known $\mathcal{T}$-piecewise constant function $\sigma_{h}$ which satisfies approximate Neumann boundary conditions. Taking the minimal choice defines an estimator (in practice an approximation will be computed)

$$
\eta_{Z}:=\min _{\tau_{h}}\left\|\sigma_{h}-\tau_{h}\right\|_{L^{2}(\Omega)},
$$

where $\tau_{h}$ is as $\sigma_{h}^{*}$ above. Then, with higher order terms that depend on the smoothness of the exact solution, we have efficiency

$$
\eta_{Z} \leq\left\|\sigma-\sigma_{h}\right\|_{L^{2}(\Omega)}+\text { h.o.t. }
$$

with a constant 1 in front of the error on the right-hand side and unknown higher order terms. (The proof of (1.15) uses the triangle inequality and an approximation estimate of $\min _{\tau_{h}}\left\|\sigma-\tau_{h}\right\|_{L^{2}(\Omega)}=$ h.o.t.)

The remaining sections of the paper are organised as follows. The detailed notation as the precise statement of the reliability, namely inequality (1.11), is introduced in Section 2. The main argument in its proof in Section 3 is a Helmholtz decomposition which allows the application of Clément approximations [Cl. In order to obtain a refined estimate we have to modify the approximation operators as in $\mathrm{Ca} 2, \mathrm{CV}, \mathrm{CB}$. The efficiency estimate (1.12) holds in a local form as shown in Section 4. The reliability of averaging techniques is established for unstructured grids in Section 5 where we indicate their efficiency. Numerical examples in Section 6 for the Stokes problem and the scheme (1.8)-(1.9) support our theoretical predictions and illustrate the superiority of the averaging technique in practise.

\section{A RELIABLE AND EFFICIENT \\ RESIDUAL-BASED A POSTERIORI ERROR ESTIMATE}

In order to state the precise form of (1.11), we specify the hypotheses on the class of conforming and nonconforming finite elements under question. 
Let $\mathcal{T}$ be a regular triangulation of $\Omega \subset \mathbb{R}^{2}$ in the sense of Ciarlet Ci], i.e., $\mathcal{T}$ is a finite partition of $\Omega$ into closed triangles or parallelograms; two distinct elements $T_{1}$ and $T_{2}$ in $\mathcal{T}$ are either disjoint or $T_{1} \cap T_{2}$ is a complete edge or a common node of both $T_{1}$ and $T_{2}$. With $\mathcal{T}$ let $\mathcal{E}$ denote the set of all edges, and we assume that $E \in \mathcal{E}$ either belongs to $\Gamma_{D}$ or $E \cap \Gamma_{D}$ has vanishing surface measure, so there is no change of boundary conditions within one edge $E \subseteq \Gamma$.

Furthermore, let $P_{k}(T)$ and $Q_{k}(T)$ denote the set of the algebraic polynomials of total and partial degree $\leq k$, respectively, and define $\mathcal{P}_{k}(T):=P_{k}(T)$ if $T$ is a triangle and $\mathcal{P}_{k}(T):=Q_{k}(T)$ if $T$ is a parallelogram.

The discrete solution $\left(u_{h}, p_{h}\right)$ satisfies (1.6)-(1.7) and is supposed to belong to $H^{2}(\mathcal{T})^{2} \times H^{1}(\mathcal{T})$, where $H^{k}(\mathcal{T}):=H^{k}\left(\bigcup_{T \in \mathcal{T}}\right.$ int $\left.T\right)$. The test function space $\mathcal{V} \times \mathcal{Q}$ in (1.6)-(1.7) is supposed to satisfy

$$
\mathcal{S}:=\mathcal{S}_{1}(\mathcal{T})^{2} \cap H_{D}^{1}(\Omega) \subseteq \mathcal{V} \subseteq H^{2}(\mathcal{T}) \quad \text { and } \quad \mathcal{L}_{0}(\mathcal{T}) \subseteq \mathcal{Q} \subset L^{2}(\Omega)
$$

Here, the Lebesgue and Sobolev spaces $L^{2}(\Omega)$ and $H^{1}(\Omega)$ are defined as usual [Ho, [M] and

$$
\begin{aligned}
\mathcal{L}_{k}(\mathcal{T}) & :=\left\{V \in L^{\infty}(\bar{\Omega}): \forall T \in \mathcal{T},\left.V\right|_{T} \in \mathcal{P}_{k}(T)\right\}, \\
\mathcal{S}_{1}(\mathcal{T}) & :=\mathcal{L}_{1}(\mathcal{T}) \cap C(\bar{\Omega}), \\
H_{D}^{1}(\Omega) & :=\left\{v \in H^{1}(\Omega)^{2}:\left.v\right|_{\Gamma_{D}}=0\right\} .
\end{aligned}
$$

Since the test and trial functions are possibly discontinuous, we define their jumps across the edges as follows. If $E \in \mathcal{E}$ is an inner edge, i.e., $E \not \subset \Gamma$, then $E=T_{1} \cap T_{2}$ for two different $T_{1}, T_{2} \in \mathcal{T}$, and $\left[\partial u_{h} / \partial s\right]$ denotes the difference of the traces of the tangential derivatives of $u_{h}$ in $T_{1}$ and $T_{2}$. Similarly $\left[\sigma_{h} n_{E}\right]$ denotes the jump of the stress vectors, i.e., for $x \in E$ and a normal $n_{E}$ on $E$,

$$
\left[\sigma_{h} n_{E}\right](x):=\lim _{\delta \rightarrow 0^{+}}\left(\sigma_{h}\left(x+\delta n_{E}\right)-\sigma_{h}\left(x-\delta n_{E}\right)\right) n_{E} .
$$

If $E \subset \Gamma_{D}$ belongs to the Dirichlet part of the boundary, then $\left[\partial u_{h} / \partial s\right]:=\partial\left(u_{D}-\right.$ $\left.u_{h}\right) / \partial s$ and $\left[\sigma_{h} n_{E}\right]:=0$. If $E \subset \bar{\Gamma}_{N}$ belongs to the Neumann part of the boundary, then $\left[\partial u_{h} / \partial s\right]:=0$ and $\left[\sigma_{h} n_{E}\right]:=g-\sigma_{h} n$.

Let $\mathcal{N}$ denote the set of all nodes in $\mathcal{T}$, and denote the set of free nodes by $\mathcal{K}:=\mathcal{N} \backslash \Gamma_{D}$. Let $\mathcal{M}$ denote the set of all midpoints of edges in $\mathcal{T}$. Let $\varphi_{z} \in \mathcal{S}^{1}(\mathcal{T})$ denote a hat function for $z \in \mathcal{N}$ defined by $\varphi_{z}(x)=0$ if $x \in \mathcal{N}$ and $x \neq z$ and $\varphi_{z}(z)=1$. Let $\omega_{z}:=\left\{x \in \Omega: \varphi_{z}(x)>0\right\}$ denote the patch of $z \in \mathcal{N}$.

For a fixed node $y \in \mathcal{N} \backslash \mathcal{K}$ we choose a neighbouring free node $\zeta(y) \in \mathcal{K}$ and set $\zeta(y):=y$ if $y \in \mathcal{K}$ such that $I(y)=\left\{z^{\prime} \in \mathcal{N}: y=\zeta\left(z^{\prime}\right)\right\}$ yields a partition $(I(z): z \in \mathcal{K})$ of $\mathcal{N}$ and the connected and open enlarged patch $\Omega_{z}:=\bigcup_{z \in I(z)} \omega_{z}$ with diameter $h_{z}:=\operatorname{diam}\left(\Omega_{z}\right)$ for $z \in \mathcal{K}$.

Theorem 2.1 implies the estimate (1.11) as a particular case.

Theorem 2.1. Let $(u, p) \in H^{1}(\Omega)^{2} \times L^{2}(\Omega)$ solve (1.1)-(1.3) and let $\left(u_{h}, p_{h}\right) \in$ $H^{2}(\mathcal{T})^{2} \times L^{2}(\Omega)$ solve (1.6)-(1.7). Suppose that $\Gamma_{D}$ is connected. Then there exist $h$-independent constants $c_{6}, \ldots, c_{9}$ that depend on the shape of the elements and the 
patches $\left(\Omega_{z}: z \in \mathcal{K}\right)$ only such that

$$
\begin{aligned}
\| \varepsilon_{\mathcal{T}}(u & \left.-u_{h}\right)\left\|_{L^{2}(\Omega)}^{2}+\right\| p-p_{h} \|_{L^{2}(\Omega)}^{2} \\
\leq & c_{6} \sum_{T \in \mathcal{T}}\left\|\operatorname{div} u_{h}\right\|_{L^{2}(T)}^{2}+c_{7} \sum_{z \in \mathcal{K}} h_{z}^{2} \min _{f_{z} \in \mathbb{R}^{2}}\left\|\operatorname{div} \sigma_{h}+f-f_{z}\right\|_{L^{2}\left(\Omega_{z}\right)}^{2} \\
& +c_{8} \sum_{E \in \mathcal{E}} h_{E}\left(\left\|\left[\sigma_{h} n_{E}\right]\right\|_{L^{2}(E)}^{2}+\left\|\left[\partial u_{h} / \partial s\right]\right\|_{L^{2}(E)}^{2}\right) \\
& +c_{9} \inf _{v}\left\|\nabla_{\mathcal{T}}\left(u_{h}-v\right)\right\|_{L^{2}(\Omega)}^{2} .
\end{aligned}
$$

The infimum in (2.2) is taken over all $v \in H^{1}(\mathcal{T})^{2}$ such that $\int_{E}[v] d s=0$ if $E \in \mathcal{E}$ and $E \not \subset \Gamma_{D}$ and $\int_{E} v d s=\int_{E} u_{D} d s$ if $E \in \mathcal{E}$ and $E \subseteq \Gamma_{D}$.

Remarks 2.1. (i) We refer to QQ for discussion and references on mixed boundary values in the Stokes problem. It seems not to be clear how a change of boundary conditions affects the regularity of the solution in the general case. As a consequence, any type of a priori estimate is avoided in Theorem 2.1 (a saturation assumption, for instance, is disputable to indicate efficiency, but not reliability).

(ii) In the case of pure Dirichlet boundary conditions, i.e., $\Gamma=\Gamma_{D}$ and $\int_{\Gamma} u_{D} \cdot n d s=0$, we normalise $p, p_{h} \in L^{2}(\Omega) / \mathbb{R}$ by $\int_{\Omega} p d x=0=\int_{\Omega} p_{h} d x$.

(iii) Our analysis is partly based on the observation of a Galerkin orthogonality for continuous test functions, i.e.,

$$
\int_{\Omega}\left(\sigma-\sigma_{h}\right): \varepsilon\left(v_{h}\right) d x=0 \quad\left(v_{h} \in \mathcal{S}\right) .
$$

To prove (2.3), we use integration by parts to infer from (1.1)-(1.3) that for $v_{h} \in \mathcal{S}$ (with $\mathcal{S}$ as in (2.1) ),

$$
\begin{aligned}
\int_{\Omega} \mathcal{A}(\varepsilon(u)): \varepsilon\left(v_{h}\right) d x-\int_{\Omega} p \operatorname{div} v_{h} d x \\
\quad=\int_{\Omega} \mathcal{A}\left(\varepsilon_{\mathcal{T}}\right)\left(u_{h}\right): \varepsilon\left(v_{h}\right) d x-\int_{\Omega} p_{h} \operatorname{div} v_{h} d x .
\end{aligned}
$$

(iv) For triangles, the condition $\mathcal{S}_{1}(\mathcal{T})^{2} \cap H_{D}^{1}(\Omega) \subseteq \mathcal{V}$ in 2.1 is satisfied for all standard conforming or nonconforming finite element spaces. On parallelograms, the nonconforming nodal basis functions do not include the conforming $Q_{1}$-finite elements; our a posteriori error estimates require nonconforming ansatz and trial spaces on parallelograms of higher order.

(v) The main argument in the proof of Theorem 2.1 is a Helmholtz decomposition which was first utilised in A. Ca1, CD, DDP in the context of a posteriori error estimates.

(vi) The refinement in Theorem 2.1 over [BB, CJ, DDP, $\mathrm{P}]$ concerns the second term on the right-hand side with the factor $c_{7}$. Since the open cover $\left(\Omega_{z}: z \in \mathcal{K}\right)$ of $\Omega$ has finite overlap, we have the estimate

$$
\sum_{z \in \mathcal{K}} h_{z}^{2} \min _{f_{z} \in \mathbb{R}^{2}}\left\|\operatorname{div} \sigma_{h}+f-f_{z}\right\|_{L^{2}\left(\Omega_{z}\right)}^{2} \leq c_{10}\left\|h_{\mathcal{T}}\left(\operatorname{div}_{\mathcal{T}} \sigma_{h}+f\right)\right\|_{L^{2}(\Omega)}^{2},
$$

where $h_{\mathcal{T}}$ is the $\mathcal{T}$-piecewise constant defined on $\Omega$ by $\left.h_{\mathcal{T}}\right|_{T}=h_{T}$ for $T \in \mathcal{T}$. If $\sigma_{h}:=\mathcal{A}\left(\varepsilon_{\mathcal{T}}\left(u_{h}\right)\right)-p_{h} I$ is $\mathcal{T}$-piecewise constant, $\operatorname{div}_{\mathcal{T}} \sigma_{h}=0$, and by using a 
Poincaré inequality we obtain

$$
\sum_{z \in \mathcal{K}} h_{z}^{2} \min _{f_{z} \in \mathbb{R}^{2}}\left\|f-f_{z}\right\|_{L^{2}\left(\Omega_{z}\right)}^{2} \leq c_{10}\left\|h_{\mathcal{T}}^{2} \nabla f\right\|_{L^{2}(\Omega)}^{2},
$$

which leads to the volume contributions in (1.10).

(vii) The term $\inf _{v}\left\|\nabla_{\mathcal{T}}\left(u_{h}-v\right)\right\|_{L^{2}(\Omega)}^{2}$ in the a posteriori estimate can be estimated once the treatment of the Dirichlet boundary conditions in the discrete problem is specified. Suppose that $\int_{E}\left[u_{h}\right] d s=0$ if $E \in \mathcal{E}$ and $E \not \subset \Gamma$ and that $u_{D}-u_{h}$ vanishes at one point of each edge. Then, with an $h$-independent constant $c_{11}>0$

$$
\inf _{v}\left\|\nabla_{\mathcal{T}}\left(u_{h}-v\right)\right\|_{L^{2}(\Omega)} \leq c_{11}\left\|h_{\mathcal{E}}^{1 / 2} \partial\left(u_{D}-u_{h}\right) / \partial s\right\|_{L^{2}\left(\Gamma_{D}\right)},
$$

from where the term $\inf _{v}\left\|\nabla_{\mathcal{T}}\left(u_{h}-v\right)\right\|_{L^{2}(\Omega)}$ is bounded by the jump terms $\left\|\left[\partial u_{h} / \partial s\right]\right\|_{L^{2}(E)}$ in $(\underline{2.2})$ and so may be neglected in the a posteriori estimate.

To see (2.7), choose $\left.v\right|_{T}:=\left.u_{h}\right|_{T}$ on each element which has no edge $E \subset \Gamma_{D}$. On each of the remaining elements $T_{m}$ we have $m \in T_{m} \cap \Gamma_{D} \cap \mathcal{M}$, where $\mathcal{M}$ denotes the set of all midpoints of edges in $\mathcal{E}$. Choose $v=u_{h}-a_{m} \psi_{m}$, where $\psi_{z}$ describes the nonconforming hat function for the midpoint $m$ on $T_{m}$. The coefficient $a_{m}=\int_{E}\left(u_{D}-u_{h}\right) d s / h_{E} \in \mathbb{R}^{2}$ guarantees that $v$ is admissible in (2.7). Then

$$
\left\|\nabla_{\mathcal{T}}\left(u_{h}-v\right)\right\|_{L^{2}(\Omega)}^{2}=\sum_{z \in \mathcal{M} \cap \Gamma_{D}}\left|a_{m}\right|^{2}\left\|\nabla \psi_{m}\right\|_{L^{2}\left(T_{m}\right)}^{2} \leq c_{11} \sum_{z \in \mathcal{M} \cap \Gamma_{D}}\left|a_{m}\right|^{2},
$$

where $c_{11}=\max _{z}\left\|\nabla \psi_{m}\right\|_{2, T_{m}}^{2}$ depends on the shape of the elements only. Since $u_{D}-u_{h}$ has a zero on $E,\left\|u_{D}-u_{h}\right\|_{2, E} \leq h_{E}\left\|\partial\left(u_{D}-u_{h}\right) / \partial s\right\|_{2, E}$ and with Cauchy's inequality,

$$
\left|a_{m}\right|^{2} \leq\left(\int_{E}\left|u_{D}-u_{h}\right| d s\right)^{2} / h_{E}^{2} \leq h_{E}\left\|\partial\left(u_{D}-u_{h}\right) / \partial s\right\|_{L^{2}(E)}^{2} .
$$

(viii) In the example of the conforming-nonconforming finite element space (1.8)(1.9), the discrete solution $u_{h}$ satisfies the Dirichlet boundary condition at the boundary nodes in the first component, i.e., $e_{1} \cdot u_{h}(z)=e_{1} \cdot u_{D}(z)$ for all $z \in \mathcal{N} \cap \Gamma_{D}$, and in the second component the discrete midpoint value equals the integral mean of the exact boundary value at a boundary edge, i.e., $e_{2} \cdot u_{h}(m)=e_{2} \cdot \int_{E} u_{D} d s / h_{E}$ for the midpoint $m$ of $E \in \mathcal{E}, E \subset \Gamma_{D}$. Then, for the same $h$-independent constant $c_{11}$ as in (2.8) the arguments in (vii) plus a finer estimate of the affine interpolation error show that the term $\inf _{v}\left\|\nabla_{\mathcal{T}}\left(u_{h}-v\right)\right\|_{L^{2}(\Omega)}^{2}$ is of higher order. Let $\mathcal{I} u_{D} \in C\left(\Gamma_{D}\right)^{2}$ denote the $\mathcal{E}$-piecewise affine nodal interpolant to $u_{D}$ on $\Gamma_{D}$. If $\left.u_{D}\right|_{E} \in H^{2}(E)$ for all $E \in \mathcal{E}$ with $E \subset \Gamma_{D}$, then

$$
\begin{gathered}
\inf _{v}\left\|\nabla_{\mathcal{T}}\left(u_{h}-v\right)\right\|_{L^{2}(\Omega)} \leq c_{11}\left\|h_{\mathcal{E}}^{1 / 2} \partial\left(u_{D}-\mathcal{I} u_{D}\right) / \partial s\right\|_{L^{2}\left(\Gamma_{D}\right)}, \\
\inf _{v}\left\|\nabla_{\mathcal{T}}\left(u_{h}-v\right)\right\|_{L^{2}(\Omega)} \leq c_{11}\left\|h_{\mathcal{E}}^{3 / 2} \partial_{\mathcal{E}}^{2} u_{D} / \partial s^{2}\right\|_{L^{2}\left(\Gamma_{D}\right)} .
\end{gathered}
$$

(ix) The constants in Theorem 2.1 depend on the shape of the patches by the overlap of $\left(\Omega_{z}: z \in \mathcal{K}\right)$. The further assumption that each element contains at least one free node reduces this dependence to usual dependence on the elements. 


\section{Proof of RELIABility}

To put emphasis on possible Neumann data, let us suppose in this section that $\Gamma_{N}$ has positive surface measure. $\left(\Gamma=\Gamma_{D}\right.$ requires the exact and discrete pressure to have a vanishing integral mean on $\Omega$, but, apart from this modification, appears less technical.)

Let $(u, p)$ solve (1.1)-(1.3), let $\left(u_{h}, p_{h}\right)$ solve (1.6)-(1.7), and consider the errors

$$
e:=u-u_{h} \in H^{1}(\mathcal{T})^{2} \quad \text { and } \quad \epsilon:=p-p_{h} \in L^{2}(\Omega) .
$$

For abbreviation, we frequently write $\|\cdot\|_{2, \Omega}:=\|\cdot\|_{L^{2}(\Omega)}$ and $\|\cdot\|_{1,2, \Omega}:=\|\cdot\|_{H^{1}(\Omega)}$ and neglect the domain $\Omega$ if there is no risk of confusion. Furthermore, we define the following residuals, which contribute to (1.10) or to the infimum in (2.2),

$$
\begin{aligned}
\eta_{1}^{2} & :=\sum_{T \in \mathcal{T}}\left\|\operatorname{div} u_{h}\right\|_{L^{2}(T)}^{2} \\
\eta_{2}^{2} & :=\sum_{z \in \mathcal{K}} h_{z}^{2} \min _{f_{z} \in \mathbb{R}^{2}}\left\|\operatorname{div} \sigma_{h}+f-f_{z}\right\|_{L^{2}\left(\Omega_{z}\right)}^{2}, \\
\eta_{3}^{2} & :=\sum_{E \in \mathcal{E}} h_{E}\left\|\left[\sigma_{h} n_{E}\right]\right\|_{L^{2}(E)}^{2}
\end{aligned}
$$

$$
\begin{array}{r}
\eta_{4}^{2}:=\inf \left\{\left\|\nabla_{\mathcal{T}}\left(u_{h}-v\right)\right\|_{L^{2}(\Omega)}^{2}: v \in H^{1}(\mathcal{T}) \text { and for all } E \in \mathcal{E}\right. \text { there holds } \\
\left.\int_{E}[v] d s=0 \text { if } E \not \subset \Gamma \text { and } \int_{E} v d s=\int_{E} u_{D} d s \text { if } E \subset \Gamma_{D}\right\},
\end{array}
$$

$$
\eta_{5}^{2}:=\sum_{E \in \mathcal{E}} h_{E}\left\|\left[\partial u_{h} / \partial s\right]\right\|_{L^{2}(E)}^{2} .
$$

In the first step of the proof, we define an auxiliary function $v$ which allows us to control the error by an energy integral.

Lemma 3.1. There exist a constant $c_{12}=c_{12}(\Omega)$ and a function $w \in H_{D}^{1}(\Omega)$ with

$$
\operatorname{div} w=\epsilon \quad \text { and } \quad\|w\|_{H^{1}(\Omega)} \leq c_{12}\|\epsilon\|_{L^{2}(\Omega)} .
$$

Furthermore, the function $v:=c_{1}^{-1} c_{2}^{2} c_{12}^{2} e-w$ satisfies (with $c_{1}, c_{2}$ from (1.4) (1.5))

$$
\frac{c_{2}^{2} c_{12}^{2}}{2}\left\|\varepsilon_{\mathcal{T}}(e)\right\|_{L^{2}(\Omega)}^{2}+\frac{1}{4}\|\epsilon\|_{L^{2}(\Omega)}^{2} \leq \int_{\Omega}\left(\sigma-\sigma_{h}\right): \varepsilon_{\mathcal{T}}(v) d x+c_{1}^{-2} c_{2}^{4} c_{12}^{4} \eta_{1}^{2} .
$$

Proof. Since $\Omega$ has a polygonal boundary, we can enlarge $\Omega$ to $\hat{\Omega}$ such that the open surface piece (or finite collection of pieces) $\Gamma_{N} \subset \partial \Omega$ belongs to the interior of the bounded Lipschitz domain $\hat{\Omega}$. The function $\epsilon$ is extended to $\hat{\epsilon}$ by a constant $\epsilon_{0}:=-\int_{\Omega} \epsilon d x / \operatorname{meas}(\hat{\Omega} \backslash \Omega)$ so that $\int_{\hat{\Omega}} \hat{\epsilon} d x=0$. The Stokes problem

$$
\Delta \hat{w}-\nabla q=0 \quad \text { and } \quad \operatorname{div} \hat{w}=\hat{\epsilon} \quad \text { in } \hat{\Omega}
$$


has a unique solution $\hat{w} \in H_{0}^{1}(\hat{\Omega})^{2}$ and $q \in L^{2}(\hat{\Omega})$, which satisfies the a priori estimate $\mathrm{GR}, \mathrm{T}, \mathrm{BF}$

$$
\|\hat{w}\|_{H^{1}(\hat{\Omega})} \leq c_{13}\|\hat{\epsilon}\|_{L^{2}(\hat{\Omega})} \leq c_{14}\|\epsilon\|_{L^{2}(\Omega)}
$$

with constants $c_{13}$ and $c_{14}$ that depend only on the geometry of $\hat{\Omega}$ and $\Omega$, but not on $\epsilon$. The restriction $w:=\left.\hat{w}\right|_{\Omega}$ satisfies (3.7).

According to the definition of $v, \sigma$, and $\sigma_{h}$, we calculate

$$
\begin{gathered}
\int_{\Omega}\left(\sigma-\sigma_{h}\right): \varepsilon_{\mathcal{T}}(v) d x=c_{1}^{-1} c_{2}^{2} c_{12}^{2} \int_{\Omega}\left(\mathcal{A}(\varepsilon(u))-\mathcal{A}\left(\varepsilon_{\mathcal{T}}\left(u_{h}\right)\right)\right): \varepsilon_{\mathcal{T}}(e) d x \\
-c_{1}^{-1} c_{2}^{2} c_{12}^{2} \int_{\Omega} \epsilon: \operatorname{div}_{\mathcal{T}} e d x-\int_{\Omega}\left(\mathcal{A}(\varepsilon(u))-\mathcal{A}\left(\varepsilon_{\mathcal{T}}\left(u_{h}\right)\right)\right): \varepsilon_{\mathcal{T}}(w) d x+\|\epsilon\|_{2, \Omega}^{2} .
\end{gathered}
$$

The symmetry of $\mathcal{A}(\varepsilon(u))-\mathcal{A}\left(\varepsilon_{\mathcal{T}}\left(u_{h}\right)\right)$ and the estimates (1.5) and (3.7) yield

$$
\begin{aligned}
& \int_{\Omega}\left(\mathcal{A}(\varepsilon(u))-\mathcal{A}\left(\varepsilon_{\mathcal{T}}\left(u_{h}\right)\right)\right): \varepsilon(w) d x \\
& \quad \leq c_{2}\left\|\varepsilon_{\mathcal{T}}(e)\right\|_{2, \Omega}\|\nabla w\|_{2, \Omega} \leq \frac{c_{2}^{2} c_{12}^{2}}{2}\left\|\varepsilon_{\mathcal{T}}(e)\right\|_{2, \Omega}^{2}+\frac{1}{2}\|\epsilon\|_{2, \Omega}^{2} .
\end{aligned}
$$

Because $\operatorname{div} u=0,\left\|\operatorname{div}_{\mathcal{T}} e\right\|_{2, \Omega}=\eta_{1}$, and (1.4), (3.11)-(3.12), we calculate

$$
\frac{c_{2}^{2} c_{12}^{2}}{2}\left\|\varepsilon_{\mathcal{T}}(e)\right\|_{2, \Omega}^{2}+\frac{1}{2}\|\epsilon\|_{2, \Omega}^{2} \leq \int_{\Omega}\left(\sigma-\sigma_{h}\right): \varepsilon_{\mathcal{T}}(v) d x+c_{1}^{-1} c_{2}^{2} c_{12}^{2} \eta_{1}\|\epsilon\|_{2, \Omega} .
$$

Now we consider a Helmholtz decomposition of $\varepsilon_{\mathcal{T}}(v)$ from CD] (see also [A] Ca1, CJ] $)$. Recall Curl $\beta=\left(\partial \beta / \partial x_{2},-\partial \beta / \partial x_{1}\right) \in L^{2}\left(\Omega ; \mathbb{R}^{d \times 2}\right)$ if $\beta \in H^{1}(\Omega)^{d}$ for $d=1,2$.

Lemma 3.2 ([CD,,$\overline{F M}])$. There exist $\alpha \in H_{D}^{1}$ and $\beta \in H^{2}(\Omega)$ with $(\operatorname{Curl} \operatorname{Curl} \beta) n=$ 0 on $\Gamma_{N}$ satisfying

$$
\varepsilon_{\mathcal{T}}(v)=\varepsilon(\alpha)+\operatorname{Curl} \operatorname{Curl} \beta \quad \text { a.e. in } \Omega .
$$

Proof. We sketch a proof for convenient reading. Let $\alpha$ solve the elliptic problem $\operatorname{div} \varepsilon(\alpha)=\operatorname{div}_{\mathcal{T}} \varepsilon_{\mathcal{T}}(v)$ with boundary conditions $\alpha=0$ on $\Gamma_{D}$ and $\varepsilon(\alpha) n=\varepsilon_{\mathcal{T}}(v) n$ on $\Gamma_{N}$, i.e., $\int_{\Omega}\left(\varepsilon(\alpha)-\varepsilon_{\mathcal{T}}(v)\right): \varepsilon(\eta) d x=0$ for all $\eta \in H_{D}^{1}(\Omega)$. Since $\varepsilon(\alpha)-\varepsilon_{\mathcal{T}}(v)$ is symmetric, the solution $\alpha$ satisfies

$$
\int_{\Omega}\left(\varepsilon(\alpha)-\varepsilon_{\mathcal{T}}(v)\right): \nabla \eta d x=0 \quad\left(\eta \in H_{D}^{1}(\Omega)^{2}\right) .
$$

Hence, each row of $\varepsilon(\alpha)-\varepsilon_{\mathcal{T}}(v)$ is divergence-free and $\left(\varepsilon(\alpha)-\varepsilon_{\mathcal{T}}(v)\right) n$ vanishes on $\Gamma_{N}$. Hence there exists some $\hat{\beta} \in H^{1}(\Omega)^{2}$ with $\varepsilon(\alpha)-\varepsilon_{\mathcal{T}}(v)=\operatorname{Curl} \hat{\beta}$ (see [GR, Sect. 3] for proofs). Moreover, since $\varepsilon(\alpha)-\varepsilon_{\mathcal{T}}(v)$ is symmetric, $\operatorname{Curl} \hat{\beta}: \varepsilon(\eta)=\operatorname{Curl} \hat{\beta}: \nabla \eta$, and by integration by parts

$$
0=\int_{\Omega} \operatorname{Curl} \hat{\beta}: \varepsilon(\eta) d x=-\int_{\Gamma_{N}} \operatorname{Curl} \hat{\beta} n \cdot \eta d s \quad\left(\eta \in H_{D}^{1}(\Omega)\right) .
$$

We conclude Curl $\hat{\beta} n=0$ whence $\partial \hat{\beta} / \partial s=0$ on $\Gamma_{N}$ and so $\hat{\beta}$ is constant on each component of $\Gamma_{N}$. If $\Gamma_{j}$ is a connectivity component of $\Gamma$ that does not include $\Gamma_{D}$, 
then $\hat{\beta}=\hat{\beta}_{j}$ is constant there and we deduce

$$
\int_{\Gamma_{j}} \hat{\beta} n d s=\hat{\beta}_{j} \int_{\Gamma_{j}} n d s=0
$$

from the divergence theorem. Since $\Gamma_{D}$ is connected, there is exactly one component $\Gamma_{0}$ that includes $\Gamma_{D}$.

The symmetry of $\operatorname{Curl} \hat{\beta}$ reads $\hat{\beta}_{2,2}=-\hat{\beta}_{1,1}$, i.e., $\operatorname{div} \hat{\beta}=0$. The divergence theorem on $\Omega$ and (3.17) then show that for all $j \neq 0$

$$
0=\int_{\Omega} \operatorname{div} \hat{\beta} d x=\int_{\Gamma_{0}} \hat{\beta} n d s .
$$

Hence, $\hat{\beta} n$ has a vanishing integral over all connectivity components of $\Gamma$ and is divergence free. Thus, there exists some $\beta \in H^{2}(\Omega) / \mathbb{R}$ that satisfies $\hat{\beta}=\operatorname{Curl} \beta$ [T, GR.

The Helmholtz decomposition (3.14) of $\varepsilon_{\mathcal{T}}(v)$ from Lemma 3.1 leads to

$$
\begin{aligned}
\frac{c_{2}^{2} c_{12}^{2}}{2}\left\|\varepsilon_{\mathcal{T}}(e)\right\|_{L^{2}(\Omega)}^{2}+\frac{1}{4}\|\epsilon\|_{L^{2}(\Omega)}^{2} & \leq \int_{\Omega}\left(\sigma-\sigma_{h}\right): \varepsilon(\alpha) d x \\
+ & \int_{\Omega}\left(\sigma-\sigma_{h}\right): \operatorname{Curl} \operatorname{Curl} \beta d x+c_{1}^{-2} c_{2}^{4} c_{12}^{4} \eta_{1}^{2} .
\end{aligned}
$$

The two integrals on the right-hand side in (3.19) will be estimated in Lemma 3.4 and 3.5 below. Therein, we require a Clément-type approximation operator [C], $\mathrm{Ci}$ ] BS] in a refined form (see also $\mathrm{CB}, \mathrm{CV}]$ ).

For a regular triangulation $\mathcal{T}$ with set of edges $\mathcal{E}$ we associate mesh-size weights $h_{\mathcal{T}}$ and $h_{\mathcal{E}}$, which are $\mathcal{T}$-piecewise and $\mathcal{E}$-piecewise constant defined on $\Omega$ and the skeleton $\bigcup \mathcal{E}$ of all points on edges by $\left.h_{\mathcal{T}}\right|_{T}=h_{T}$ for $T \in \mathcal{T}$ and $\left.h_{\mathcal{E}}\right|_{E}=h_{E}$ for $E \in \mathcal{E}$, respectively.

Lemma 3.3 (Ca2 ). There exists a linear mapping $\mathcal{J}: H_{D}^{1}(\Omega)^{2} \rightarrow \mathcal{S}$, bounded if domain and range space are endowed with $H^{1}$-semi norms, which satisfies

$$
\left\|h_{\mathcal{T}}^{-1}(\varphi-\mathcal{J} \varphi)\right\|_{L^{2}(\Omega)}+\left\|h_{\mathcal{E}}^{-1 / 2}(\varphi-\mathcal{J} \varphi)\right\|_{L^{2}(\bigcup \mathcal{E})} \leq c_{15}\|\nabla \varphi\|_{L^{2}(\Omega)}
$$

for all $\varphi \in H_{D}^{1}(\Omega)$. In addition, there holds for all $R \in L^{2}(\Omega)^{2}$

$$
\int_{\Omega} R \cdot(\varphi-\mathcal{J} \varphi) d x \leq c_{16}\|\nabla \varphi\|_{L^{2}(\Omega)}\left(\sum_{z \in \mathcal{K}} h_{z}^{2} \min _{R_{z} \in \mathbb{R}^{2}} \int_{\Omega_{z}}\left|R-R_{z}\right|^{2} d x\right)^{1 / 2} .
$$

The positive constants $c_{15}, c_{16}$ do not depend on the mesh-sizes $h_{\mathcal{T}}$ and $h_{\mathcal{E}}$, but on the shape of the elements only.

The first integral on the right-hand side in (3.19) is studied in the next lemma.

Lemma 3.4. We have

$$
\int_{\Omega}\left(\sigma-\sigma_{h}\right): \varepsilon(\alpha) d x \leq \max \left\{c_{15}, c_{16}\right\}\left(\eta_{2}^{2}+\eta_{3}^{2}\right)^{1 / 2}\|\nabla \alpha\|_{L^{2}(\Omega)} .
$$


Proof. Utilising (2.3), $A:=\mathcal{J} \alpha \in \mathcal{S} \subset \mathcal{V}, \alpha-A \in H_{D}^{1}(\Omega)$, and elementwise integration by parts, we infer

$$
\begin{aligned}
\int_{\Omega}\left(\sigma-\sigma_{h}\right): \varepsilon(\alpha) d x & =\int_{\Omega}\left(\sigma-\sigma_{h}\right): \varepsilon(\alpha-A) d x \\
& =\int_{\Omega}\left(\operatorname{div} \sigma_{h}+f\right) \cdot(\alpha-A) d x+\sum_{E \in \mathcal{E}} \int_{E}\left[\sigma_{h} n_{E}\right](\alpha-A) d s .
\end{aligned}
$$

Recall that $\left[\sigma_{h} n_{E}\right]$ is the jump of $\sigma_{h} n_{E}$ across an interior element boundary $E \in \mathcal{E}$, and is defined by $g-\sigma_{h} n$ on $\Gamma_{N}$ and $\left[\sigma_{h} n_{E}\right]=0$ on $\Gamma_{D}$. From Cauchy's inequality and (3.20)-(3.21) we conclude

$$
\begin{aligned}
\int_{\Omega}(\sigma & \left.-\sigma_{h}\right): \varepsilon_{\mathcal{T}}(\alpha) d x \\
& \leq\left(c_{16} \sum_{z \in \mathcal{K}} h_{z}^{2} \min _{f_{z} \in \mathbb{R}^{2}}\left\|\operatorname{div} \sigma_{h}+f-f_{z}\right\|_{2, \Omega_{z}}^{2}+c_{15} \sum_{E \in \mathcal{E}} h_{E}\left\|\left[\sigma_{h} n_{E}\right]\right\|_{2, E}^{2}\right)^{1 / 2}\|\nabla \alpha\|_{2, \Omega}^{2} \\
& \leq \max \left\{c_{15}, c_{16}\right\}\left(\eta_{2}^{2}+\eta_{3}^{2}\right)^{1 / 2}\|\nabla \alpha\|_{2, \Omega} . \quad \square
\end{aligned}
$$

The second integral on the right-hand side in (3.19) is studied in the next lemma, where $c_{17}$ will be characterised below in (3.28) as an analogue to $c_{15}$.

Lemma 3.5. We have

$$
\int_{\Omega}\left(\sigma-\sigma_{h}\right): \operatorname{Curl} \operatorname{Curl} \beta d x \leq c_{1}^{-1} c_{2}^{2} c_{12}^{2} c_{17}\left(\eta_{4}^{2}+\eta_{5}^{2}\right)^{1 / 2}\left\|\sigma-\sigma_{h}\right\|_{L^{2}(\Omega)} .
$$

Proof. Let $a \in H_{D}^{1}(\Omega)$ and $b \in H^{2}(\Omega)$ define a Helmholtz decomposition of $\sigma-\sigma_{h}$ as in Lemma 3.2 i.e.,

$$
\sigma-\sigma_{h}=\varepsilon(a)+\operatorname{Curl} \operatorname{Curl} b \quad \text { a.e. in } \Omega .
$$

Since $(\operatorname{Curl} \operatorname{Curl} b) n=0$ on $\Gamma_{N}$ we have $L^{2}$-orthogonality of Curl Curl $\beta$ and $\varepsilon(a)$ and deduce from $v:=c_{1}^{-1} c_{2}^{2} c_{12}^{2} e-w$ and an integration by parts that

$$
\begin{aligned}
c_{1} c_{2}^{-2} c_{12}^{-2} \int_{\Omega}\left(\sigma-\sigma_{h}\right): \operatorname{Curl} \operatorname{Curl} \beta d x=\int_{\Omega} \operatorname{Curl} \operatorname{Curl} b: \varepsilon_{\mathcal{T}}(e) d x \\
=\int_{\Gamma_{D}} u_{D} \cdot \operatorname{Curl} \operatorname{Curl} b n d s-\int_{\Omega} \operatorname{Curl} \operatorname{Curl} b: \varepsilon_{\mathcal{T}}\left(u_{h}\right) d x .
\end{aligned}
$$

Let $B \in \mathcal{S}_{1}(\mathcal{T})^{2}$ denote an approximation to Curl $b$ as in Lemma 3.3 where the role of the Dirichlet and Neumann boundaries is interchanged; here $\Gamma_{N}$ acts as the Dirichlet boundary, i.e., $B \in C(\bar{\Omega})^{2}$, Curl $b=B$ on $\Gamma_{N}$. Recall that $0=$ Curl Curl $b n=\partial \operatorname{Curl} b / \partial s$ such that $\operatorname{Curl} b$ is constant on each component of $\Gamma_{N}$ and so the interpolation indeed yields $\operatorname{Curl} b=B$ on $\Gamma_{N}$. As in Lemma 3.3 we have

$$
\|\operatorname{Curl} B\|_{2}+\left\|h_{\mathcal{E}}^{-1 / 2}(\operatorname{Curl} b-B)\right\|_{2, \cup \mathcal{E}} \leq c_{17}\left\|D^{2} b\right\|_{2} .
$$


Note that $\operatorname{Curl} B n_{E}=0$ on $\Gamma_{N}$ and, furthermore, $\operatorname{Curl} B n_{E}$ is constant on each $E \in \mathcal{E}$. Thus, for $v \in H^{1}(\mathcal{T})^{2}$ as in Theorem 2.1,

$$
\begin{aligned}
\int_{\Gamma_{D}} u_{D} \cdot \operatorname{Curl} B n d s & =\int_{\Gamma_{D}} v \cdot \operatorname{Curl} B n d s \\
& +\sum_{E \in \mathcal{E}} \int_{E \backslash \Gamma}[v] \cdot \operatorname{Curl} B n_{E} d s=\int_{\Omega} \nabla_{\mathcal{T}} v: \operatorname{Curl} B d x
\end{aligned}
$$

where we utilised an elementwise integration by parts. From the symmetry of Curl Curl $b$, (3.27), and (3.29), we infer

$$
\begin{aligned}
c_{1} c_{2}^{-2} c_{12}^{-2} & \int_{\Omega}\left(\sigma-\sigma_{h}\right): \operatorname{Curl} \operatorname{Curl} \beta d x=\int_{\Gamma_{D}} u_{D} \cdot \operatorname{Curl}(\operatorname{Curl} b-B) n d s \\
& +\int_{\Omega} \operatorname{Curl}(B-\operatorname{Curl} b): \nabla_{\mathcal{T}} u_{h} d x-\int_{\Omega} \operatorname{Curl} B: \nabla_{\mathcal{T}}\left(u_{h}-v\right) d x .
\end{aligned}
$$

From Curl $b=B$ on $\Gamma_{N}$ and the integration by parts formula on the closed Lipschitz curve (or curves of) $\Gamma$ we deduce

$$
\begin{aligned}
\int_{\Gamma_{D}} u_{D} \cdot \operatorname{Curl}(\operatorname{Curl} b-B) n d s=\int_{\Gamma_{D}} & u_{D} \cdot \partial(\operatorname{Curl} b-B) / \partial s d s \\
& =-\int_{\Gamma_{D}} \partial u_{D} / \partial s \cdot(\operatorname{Curl} b-B) d s .
\end{aligned}
$$

Elementwise integration by parts, Cauchy's inequality, (3.28), (3.30), and (3.31) result in

$$
\begin{aligned}
& c_{1} c_{2}^{-2} c_{12}^{-2} \int_{\Omega}\left(\sigma-\sigma_{h}\right): \operatorname{Curl} \operatorname{Curl} \beta d x \leq\|\operatorname{Curl} B\|_{2}\left\|\nabla_{\mathcal{T}}\left(u_{h}-v\right)\right\|_{2} \\
& +\int_{\cup \mathcal{E}}\left[\partial u_{h} / \partial s\right] \cdot(\operatorname{Curl} b-B) d s \leq c_{17}\left\|D^{2} b\right\|_{2} \max \left\{\eta_{5},\left\|\nabla_{\mathcal{T}}\left(u_{h}-v\right)\right\|_{2}\right\} .
\end{aligned}
$$

Proof of Theorem 2.1. Combining Lemmas 3.4 and 3.5 we obtain from (3.19) and $\left\|\sigma-\sigma_{h}\right\|_{2} \leq c_{2}\left\|\varepsilon_{\mathcal{T}}(e)\right\|_{2}+\|\epsilon\|_{2}$ and $\|\varepsilon(\alpha)\|_{2} \leq\left\|\varepsilon_{\mathcal{T}}(e)\right\|_{2}$ that

$$
\begin{aligned}
& \frac{c_{2}^{2} c_{12}^{2}}{2}\left\|\varepsilon_{\mathcal{T}}(e)\right\|_{2}^{2}+\frac{1}{4}\|\epsilon\|_{2}^{2} \leq \max \left\{c_{15}, c_{16}\right\}\left(\eta_{2}^{2}+\eta_{3}^{2}\right)^{1 / 2}\left\|\varepsilon_{\mathcal{T}}(e)\right\|_{2} \\
& \quad+c_{1}^{-1} c_{2}^{2} c_{12}^{2} c_{17}\left(\eta_{4}^{2}+\eta_{5}^{2}\right)^{1 / 2}\left(c_{2}\left\|\varepsilon_{\mathcal{T}}(e)\right\|_{2}+\|\epsilon\|_{2}\right)+c_{1}^{-2} c_{2}^{4} c_{12}^{4} \eta_{1}^{2}
\end{aligned}
$$

With Young's inequality, the terms $\left\|\varepsilon_{\mathcal{T}}(e)\right\|_{2}$ and $\|\epsilon\|_{2}$ on the left-hand side can be absorbed. This concludes the proof of Theorem 2.1.

\section{EFFICIENCY}

This section is devoted to efficiency investigations whose aim is to prove the converse estimate up to higher order terms. Some of the technical results of the section are preliminary to the proof of reliability of the averaging techniques in the subsequent section. 


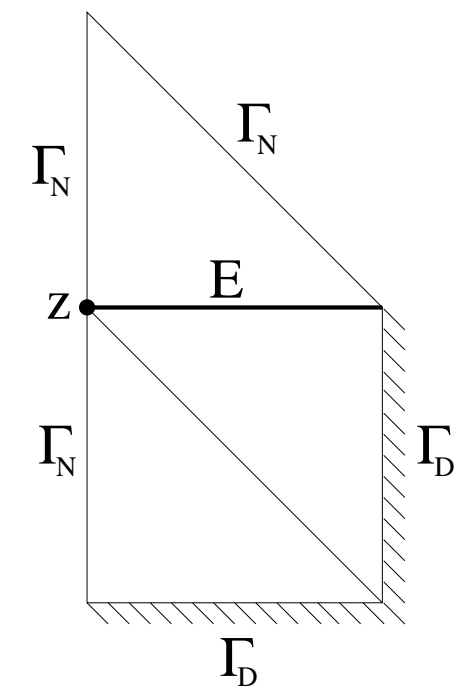

Figure 1. Coarse triangulation where the inner edge $E$ violates geometric restrictions

To indicate the efficiency of the a posteriori error bound (1.10) we follow Verfürth V1 and refine a corresponding estimate in DDP].

To cover the conforming-nonconforming scheme (1.8)-(1.9) as well as conforming finite element schemes, we suppose that $u_{h}$ belongs to $\mathcal{W}=\mathcal{W}_{1} \times \mathcal{W}_{2}$ where the jumps on interior element edges or Dirichlet edges satisfy different continuity conditions in each component:

$$
\begin{aligned}
& \mathcal{W}_{1}:=\left\{V \in \mathcal{L}_{k}(\mathcal{T}): \forall E \in \mathcal{E},[V] \text { vanishes at the endpoints of } E\right\} \\
& \mathcal{W}_{2}:=\left\{V \in \mathcal{L}_{k}(\mathcal{T}): \forall E \in \mathcal{E}, \int_{E}[V] d s=0\right\}
\end{aligned}
$$

Here, $1 \leq k$ and the jump on boundary edges is understood as $[V]:=0$ on $\bar{\Gamma}_{N}$ and $[V]:=u_{D}-V$ on $\Gamma_{D}$.

According to the different role of the two components, we need different mild restrictions on the coarseness of the mesh: Assume first that each connectivity component of $\Gamma_{D}$ ( $\Gamma_{D}$ is no longer necessarily connected) contains more than one edge in $\mathcal{E}$. Second assume that each edge $E \not \subset \Gamma$ has at least one endpoint which is an interior node (see Figure 1 where the second condition is violated).

Suppose that $u_{D} \in C\left(\Gamma_{D}\right)^{2}$ satisfies $\left.u_{D}\right|_{E} \in H^{1}(E)^{2}$ for all $E \in \mathcal{E}$ with $E \subset \Gamma_{D}$, and write $\partial_{\mathcal{E}}^{m} u_{D} / \partial s^{m}$ for the $\mathcal{E}$-piecewise derivative of $u_{D}$ on $\Gamma_{D}$ with respect to the arc-length. Recall that $\mathcal{I} u_{D} \in C\left(\Gamma_{D}\right)^{2}$ denotes the $\mathcal{E}$-piecewise affine nodal interpolant to $u_{D}$ on $\Gamma_{D}$ (i.e., $\mathcal{I} u_{D}(z)=u_{D}(z)$ for all $z \in \mathcal{N} \cap \Gamma_{D}$, and $\left.\mathcal{I} u_{D}\right|_{E}$ is affine on $E \in \mathcal{E}$ with $\left.E \subset \Gamma_{D}\right)$. Let $f_{\mathcal{T}} \in \mathcal{S}_{0}(\mathcal{T})^{2}$ be the $L^{2}$-projection of $f$ on $\mathcal{S}_{0}(\mathcal{T})^{2}$ and let $g_{\mathcal{E}}$ denote an $\mathcal{E}$-piecewise polynomial approximation to $g$ on $\Gamma_{N}$.

Theorem 4.1 implies the estimate (1.12) as a particular case.

Theorem 4.1. Let $(u, p) \in H^{1}(\Omega)^{2} \times L^{2}(\Omega)$ solve (1.1) $-(1.3),\left(u_{h}, p_{h}\right) \in H^{2}(\mathcal{T})^{2} \times$ $L^{2}(\Omega)$ solve (1.6)-(1.7), $u_{h} \in \mathcal{W}$, and set $\sigma_{h}:=\mathcal{A}\left(\varepsilon_{\mathcal{T}}\left(u_{h}\right)\right)-p_{h} I$. Then there exists an $h$-independent constant $c_{19}>0$ such that, for all $T \in \mathcal{T}$ and the patch 
$\omega_{T}:=\bigcup\left\{T^{\prime} \in \mathcal{T}: T \cap T^{\prime} \in \mathcal{E} \cup \mathcal{T}\right\}$ of neighbouring elements

$$
\begin{aligned}
\sum_{E \in \mathcal{E} \wedge E \subset \partial T} & h_{E}\left(\left\|\left[\sigma_{h} n_{E}\right]\right\|_{L^{2}(E)}^{2}+\left\|\left[\partial u_{h} / \partial s\right]\right\|_{L^{2}(E)}^{2}\right) \\
\leq c_{19}\left(\inf _{v=u_{D} \text { on } \Gamma_{D}}\left\|\varepsilon_{\mathcal{T}}\left(u_{h}-v\right)\right\|_{L^{2}\left(\omega_{T}\right)}^{2}\right. & \quad+\inf _{\tau \in H\left(\operatorname{div} ; \omega_{T}\right)^{2}}\left(\left\|\sigma_{h}-\tau\right\|_{2, \omega_{T}}^{2}+h_{E}^{2}\left\|\operatorname{div}_{\mathcal{T}}\left(\sigma_{h}-\tau\right)\right\|_{2, \omega_{T}}^{2}\right) \\
& +\left\|h_{\mathcal{E}}^{1 / 2}\left(g-g_{\mathcal{E}}\right)\right\|_{L^{2}\left(\Gamma_{N} \cap \partial \omega_{T}\right)}^{2}+h_{T}^{2}\left\|f-f_{\mathcal{T}}\right\|_{L^{2}\left(\omega_{T}\right)}^{2} \\
& \left.+\left\|h_{\mathcal{E}}^{1 / 2} \partial\left(u_{D}-\mathcal{I} u_{D}\right) / \partial s\right\|_{L^{2}\left(\Gamma_{D} \cap \partial \omega_{T}\right)}^{2}\right) .
\end{aligned}
$$

In particular, we have the efficiency estimate

$$
\begin{aligned}
& \sum_{E \in \mathcal{E} \wedge E \subset \partial T} h_{E}\left(\left\|\left[\sigma_{h} n_{E}\right]\right\|_{L^{2}(E)}^{2}+\left\|\left[\partial u_{h} / \partial s\right]\right\|_{L^{2}(E)}^{2}\right) \\
& \quad+h_{T}^{2}\left\|f+\operatorname{div} \sigma_{h}\right\|_{L^{2}(T)}^{2}+\left\|\operatorname{div} u_{h}\right\|_{L^{2}(T)}^{2} \\
& \leq c_{19}\left(\left\|\varepsilon_{\mathcal{T}}\left(u-u_{h}\right)\right\|_{L^{2}\left(\omega_{T}\right)}^{2}+\left\|p-p_{h}\right\|_{L^{2}\left(\omega_{T}\right)}^{2}+\left\|h_{\mathcal{E}}^{1 / 2}\left(g-g_{\mathcal{E}}\right)\right\|_{L^{2}\left(\Gamma_{N} \cap \partial \omega_{T}\right)}^{2}\right. \\
& \left.\quad+h_{T}^{2}\left\|f-f_{\mathcal{T}}\right\|_{L^{2}\left(\omega_{T}\right)}^{2}+\left\|h_{\mathcal{E}}^{1 / 2} \partial\left(u_{D}-\mathcal{I}_{D}\right) / \partial s\right\|_{L^{2}\left(\Gamma_{D} \cap \partial \omega_{T}\right)}^{2}\right)
\end{aligned}
$$

Remarks 4.1. (i) The constant $c_{19}$ in Theorem 4.1 depends on the shape and on the degree of the finite elements (not on their diameters).

(ii) The condition $u_{h} \in \mathcal{W}$ is satisfied for all conforming finite element methods as well as for the conforming-nonconforming scheme (1.6)-(1.7).

(iii) The compatibility condition in (4.1) could be further relaxed. The proof shows that any compatibility condition which guarantees an affine function $e_{1} \cdot[v]$ vanishes is indeed sufficient.

(iv) The mild restrictions on the mesh are violated in the example indicated in Figure 1 Note that successive red-refinements cannot change that the top triangle can rigidly move around the midpoint of $E$. A green-refinement of $E$ in the top triangle cures the failure (see, e.g., [V1] for the definition of red-green-blue refinement).

(v) In corresponding results in [BB, CJ] the error term $\left\|\nabla\left(u-u_{h}\right)\right\|_{2, \Omega_{T}}$ arises which is replaced here by the Green strain error $\left\|\varepsilon\left(u-u_{h}\right)\right\|_{2, \Omega_{T}}$. For conforming schemes, $u-u_{h} \in H_{D}^{1}(\Omega)$ and this improvement is not important since Korn's inequality provides global equivalence. For nonconforming schemes, Korn's inequality is not available [FM]. For the conforming-nonconforming finite element scheme with (1.8)-(1.9), Korn's inequality is globally available for $u_{h}$ and $u$ but not necessarily for $u-u_{h}$. (The different statement in $[\mathrm{BB}]$ is still unproven.)

The remaining part of the section is devoted to the proof of Theorem4.1preceded by several technical lemmas.

The first lemma provides a version of Korn's inequality (certainly known to the experts but not easily found in textbooks).

Lemma 4.1. Let $\mathcal{R} \mathcal{M}(\Omega)$ denote the rigid body motions in $\mathbb{R}^{d}(d=2,3)$. Then, $\|\cdot\|_{H^{1}(\Omega)}$ and $\|\varepsilon(\cdot)\|_{L^{2}(\Omega)}$ are two equivalent norms on $H^{1}(\Omega)^{d} / \mathcal{R} \mathcal{M}(\Omega)$. 
Proof. The standard version of Korn's inequality is the estimate

$$
\|\cdot\|_{2}+\|\nabla \cdot\|_{2} \leq c_{20}\left(\|\cdot\|_{2}+\|\varepsilon(\cdot)\|_{2}\right) \text { in } H^{1}(\Omega)^{d}
$$

and can be found in textbooks. The main point in the lemma is the related estimate

$$
\min _{r \in \mathcal{R} \mathcal{M}(\Omega)}\|v-r\|_{1,2} \leq c_{12}\|\varepsilon(v)\|_{2}
$$

for $v \in H^{1}(\Omega)^{d}$. We sketch an indirect proof for convenient reading. If this inequality was false, we could find a sequence $\left(w_{j}\right)$ in $H^{1}(\Omega)^{2} / \mathcal{R} \mathcal{M}(\Omega)$ with

$$
\lim _{j \rightarrow \infty}\left\|\varepsilon\left(w_{j}\right)\right\|_{2}=0 \quad \text { and } \min _{r \in \mathcal{R} \mathcal{M}}\left\|w_{j}-r\right\|_{1,2}=1 .
$$

Banach-Alaoglu's theorem yields a weakly convergent subsequence in $H^{1}(\Omega)^{d}$ and by Rellich's theorem there exists a strongly convergent subsequence $\left(v_{j}\right)$ in $L^{2}(\Omega)^{d}$. Hence we may and will assume without loss of generality that there exists a weak limit $v$ in $H^{1}(\omega)^{d}$ with $\lim _{j \rightarrow \infty}\left\|v-v_{j}\right\|_{2}=0$. Since $\|\varepsilon(\cdot)\|_{2}$ is sequentially weakly lower semi-continuous, we deduce $\varepsilon(v)=0$, i.e., $v \in \mathcal{R} \mathcal{M}(\Omega)$. Korn's inequality (4.5) shows

$$
1=\min _{r \in \mathcal{R} \mathcal{M}(\Omega)}\left\|v_{j}-r\right\|_{1,2} \leq\left\|\nabla\left(v_{j}-v\right)\right\|_{1,2} \leq c_{20}\left(\left\|\varepsilon\left(v_{j}-v\right)\right\|_{2}+\left\|v_{j}-v\right\|_{2}\right),
$$

but the right-hand side in (4.7) tends toward zero. This contradiction proves (4.6). We omit the proof of the remaining assertions.

Lemma 4.2. Let $T_{1}, \ldots, T_{J} \in \mathcal{T}$ be a sequence of neighbouring elements such that $E=T_{j} \cap T_{j+1} \in \mathcal{E}$ is an edge which is not parallel to the $x_{1}$-axis. Then, all $r \in \mathcal{W}$ with $r_{T_{j}} \in \mathcal{R} \mathcal{M}\left(T_{j}\right), j=1, \ldots, J$ are rigid, i.e., $r \in \mathcal{R} \mathcal{M}\left(\bigcup_{j=1}^{J} T_{j}\right)$.

Proof. It suffices to prove the assertion for $J=2$; the general case follows by induction. Consider a common edge $E$ of two distinct neighbouring elements $T_{1}$ and $T_{2}$ where there exist real numbers $a_{T}, b_{T}, c_{T}$ with

$$
r(x)=\left(a_{T}-c_{T} x_{2}, b_{T}+c_{T} x_{1}\right) \quad \text { for } x \in T .
$$

The condition (4.1) shows that the first component $\left[r_{1}\right]$ of the jump $[r]$ of $r$ vanishes at two distinct points. In case $E$ is not aligned with the $x_{1}$-axis, this shows that the affine function $\left[r_{1}\right]$ vanishes, i.e., $a_{T_{1}}=a_{T_{2}}$ and $c_{T_{1}}=c_{T_{2}}$. Condition (4.2) implies that $\left[r_{2}\right]$ vanishes at the midpoint of $E$. Knowing $c_{T_{1}}=c_{T_{2}}$ already, $b_{T_{1}}=b_{T_{2}}$ follows from this and (4.8). Thus, if $E$ is not aligned with the $x_{1}$-axis, $[r]=0$, i.e., $r \in \mathcal{R} \mathcal{M}\left(T_{1} \cup T_{2}\right)$.

Lemma 4.3. Let $\omega_{z}$ be the patch of $z \in \mathcal{N}$. Suppose that either $z$ is an interior point of $\Omega$ or that $z$ does not belong to exactly one edge $E \in \mathcal{E}$ parallel to the $x_{1}$-axis (cf. Figure 11). Let $r \in \mathcal{W}$ such that $\left.r\right|_{T} \in \mathcal{R} \mathcal{M}(T)$ for all $T \in \mathcal{T}$ with $T \subset \overline{\omega_{z}}$. Then $\left.r\right|_{\omega_{z}} \in \mathcal{R} \mathcal{M}\left(\omega_{z}\right)$.

Proof. In two dimensions, there are either zero, one or two edges $E \sqsubset \omega_{z}$ with $E \subset \mathbb{R} \times\left\{z_{2}\right\}$, where " $E \sqsubset \omega_{z}$ " stands for all edges $E \in \mathcal{E}$ with $z \in E$. If there is no such edge, the assertion follows from Lemma 4.2 ,

In case there is one such edge $E \subset \mathbb{R} \times\left\{z_{2}\right\}$ and $z$ is an interior point, Lemma 4.2 reveals $r \in \mathcal{R} \mathcal{M}\left(\omega_{z} \backslash\{E\}\right)$ and, since the set $\omega_{z} \backslash\{E\}$ is connected, $r \in \mathcal{R} \mathcal{M}\left(\omega_{z}\right)$.

In case there are two such edges $E_{1}$ and $E_{2} \subset \mathbb{R} \times\left\{z_{2}\right\}$ we find that $r$ is a rigid body motion on either of the two components of $\omega_{z} \backslash \mathbb{R} \times\left\{z_{2}\right\}$. The jump $\left[r_{2}\right]$ across 
$E_{j} \subset \mathbb{R} \times\left\{z_{2}\right\}$ is affine on $\mathbb{R} \times\left\{z_{2}\right\}$ and vanishes at the midpoints of $E_{j}$. Hence, $\left[r_{2}\right]=0$. From this and $\left[r_{1}\right]=0$ we then deduce $r \in \mathcal{R} \mathcal{M}\left(\omega_{z}\right)$.

Lemma 4.4. Let $\omega_{z}$ be a patch of $z \in \mathcal{N} \cap \Gamma_{D}$ such that $\Gamma_{D} \cap \partial \omega_{z}=E_{1} \cup E_{2}$ for two distinct edges $E_{1}, E_{2} \in \mathcal{E}$ which are parallel. Suppose $u_{D}=0$ and $r \in \mathcal{W}$ with $\varepsilon_{\mathcal{T}}(r)=0$ on $\omega_{z}$. Then $r=0$ on $\omega_{z}$.

Proof. Lemma 4.2 shows that $r$ is a rigid body motion on each connectivity component of $\omega_{z} \backslash \mathbb{R} \times\left\{z_{2}\right\}$. If there is no edge $E$ with $z \in E$ parallel to the $x_{1}$-axis, then $r$ is a global rigid body motion which is zero at the midpoint $m_{j}$ of $E_{j}$ and so $r=0$. The same conclusion can be drawn if $E_{1}$ and $E_{2}$ are parallel to the $x_{1}$-axis. In the remaining case, $E_{j}$ is not parallel to the $x_{1}$-axis but possibly one other edge. As in the proof of Lemma 4.2 we deduce from the boundary conditions in $\mathcal{W}$ that $\left.r\right|_{E_{j}}=0$. Hence, $r=0$ on that component of $\omega_{z} \backslash \mathbb{R} \times\left\{z_{2}\right\}$, to which $E_{j}$ belongs. $E_{1}$ and $E_{2}$ belong to different components and so $r=0$ on $\omega_{z}$.

Lemma 4.5. Let $\omega_{z}$ be a patch of $z \in \mathcal{N}$ which is either an interior node or belongs to a straight part of $\Gamma_{D}$ in the sense that $\{z\}=E_{1} \cap E_{2}, E_{1}, E_{2} \in \mathcal{E}$ for parallel $E_{1}, E_{2} \subset \Gamma_{D}$. Then, there exists an h-independent constant $c_{22}>0$ such that for all $v_{h} \in \mathcal{W}$,

$$
\begin{aligned}
& \left(\sum_{E \sqsubset \omega_{z}} h_{E}\left\|\left[\partial v_{h} / \partial s\right]\right\|_{L^{2}(E)}^{2}\right)^{1 / 2} \\
\leq & c_{22}\left(\left\|h_{\mathcal{E}}^{1 / 2} \partial\left(u_{D}-\mathcal{I} u_{D}\right) / \partial s\right\|_{L^{2}\left(\Gamma_{D} \cap \partial \omega_{z}\right)}+\inf _{v=u_{D} \text { on } \Gamma_{D}}\left\|\varepsilon\left(v_{h}-v\right)\right\|_{L^{2}\left(\omega_{z}\right)}\right) .
\end{aligned}
$$

In the infimum, " $v=u_{D}$ on $\Gamma_{D}$ " stands for all $v \in H^{1}\left(\omega_{z}\right)^{2}$ if $z$ is an interior node and otherwise for all $v \in H^{1}\left(\omega_{z}\right)^{2}$ with $\left.v\right|_{E_{1} \cup E_{2}}=\left.u_{D}\right|_{E_{1} \cup E_{2}}$.

Proof. In the first step, we prove the lemma for the homogeneous case $u_{D}=0$. The left- and right-hand sides of (4.9) are semi-norms on the spaces

$$
\mathcal{C}_{z}:=\mathcal{W}_{z} \cap H_{D}^{1}\left(\omega_{z}\right) \subset \mathcal{W}_{z}:=\left\{\left.V\right|_{\omega_{z}}: V \in \mathcal{W}\right\},
$$

where $H_{D}^{1}\left(\omega_{z}\right):=H^{1}\left(\omega_{z}\right)^{2}$ if $z$ is an interior node and $H_{D}^{1}\left(\omega_{z}\right):=\left\{v \in H^{1}\left(\omega_{z}\right)^{2}\right.$ : $v=0$ on $\left.E_{1} \cup E_{2}\right\}$ if $z \in E_{1} \cup E_{2} \subset \Gamma_{D}$. We claim that the right-hand side of (4.9) is a norm on $\mathcal{W}_{z} / \mathcal{C}_{z}$.

Suppose that $v_{h} \in \mathcal{W}_{z}$ satisfies $\inf _{v \in H_{D}^{1}\left(\omega_{z}\right)}\left\|\varepsilon_{\mathcal{T}}\left(v_{h}-v\right)\right\|_{L^{2}\left(\omega_{z}\right)}=0$. Then, there exists a sequence $\left(v_{j}\right)$ in $H_{D}^{1}\left(\omega_{z}\right)$ with $\lim _{j \rightarrow \infty}\left\|\varepsilon_{\mathcal{T}}\left(v_{h}-v_{j}\right)\right\|_{L^{2}\left(\omega_{z}\right)}=0$. Hence, $\varepsilon\left(v_{j}\right)$ is bounded in $L^{2}\left(\omega_{z}\right)$ and so is $\left(v_{j}\right)$ in $H_{D}^{1}\left(\omega_{z}\right) / \mathcal{R} \mathcal{M}\left(\omega_{z}\right)$ owing to Korn's inequality in the form of Lemma 4.1. Banach-Alaoglu's theorem yields a weakly convergent subsequence $\left(v_{k}\right)$ with weak limit $v$ in $H^{1}(\Omega)^{2}$. Then $\left\|\varepsilon_{\mathcal{T}}\left(v_{h}-v\right)\right\|_{2, \omega_{z}}=$ 0 , and so $r:=v_{h}-v$ belongs to $\mathcal{R M}(T)$ for all $T \in \mathcal{T}$ with $T \subset \overline{\omega_{z}}$. Note that $v=v_{h}-r$ is a piecewise polynomial in $H_{D}^{1}\left(\omega_{z}\right)$ and so $v \in \mathcal{C}_{z}$ and $r \in \mathcal{W}_{z}$.

For $z \notin \Gamma_{D}$, Lemma 4.3 shows that $r$ is a rigid body motion on $\omega_{z}$ and this implies $v_{h}=r+v \in \mathcal{C}_{z}$. If $z$ lies on the Dirichlet boundary, Lemma 4.4 shows $r=0$ and so $v_{h} \in \mathcal{C}_{z}$. Thus in any case $v_{h}=0$ in the quotient space $\mathcal{W}_{z} / \mathcal{C}_{z}$.

The left- and right-hand sides of (4.9) are norms on the finite-dimensional space $\mathcal{W}_{z} / \mathcal{C}_{z}$ and hence equivalent. This proves (4.9) with a constant $c_{22}$ that depends on $\omega_{z}$ provided $u_{D}=0$. A scaling argument shows that the weights $h_{E}$ are chosen properly so that $c_{22}$ is independent from $h_{z}$ but merely dependent on the shape of the elements and so the shape of the patch. 
In the second part of the proof, we have $z \in \Gamma_{D} \cap E_{1} \cap E_{2}$ and allow $u_{D} \neq 0$. We extend the nodal interpolant $\mathcal{I} u_{D}$ to $\Omega$ by taking the remaining nodal values equal to zero. Then, $\mathcal{I} u_{D} \in \mathcal{S}^{1}(\mathcal{T})^{2}$. Let $\psi_{j}$ be the nonconforming nodal basis function related to the midpoint $m_{j}$ of the edge $E_{j}$. Then the discrete function

$$
\begin{aligned}
w_{h} & :=v_{h}-\mathcal{I} u_{D}-a_{1} \psi_{1} e_{2}-a_{2} \psi_{2} e_{2}, \\
a_{j} & :=\frac{e_{2}}{h_{E_{j}}} \cdot \int_{E_{j}}\left(u_{D}-\mathcal{I} u_{D}\right) d s, \quad j=1,2,
\end{aligned}
$$

satisfies the compatibility conditions for homogeneous Dirichlet data as considered in the first step of this proof. Hence, we obtain in particular

$$
h_{E_{j}}^{1 / 2}\left\|\partial w_{h} / \partial s\right\|_{2, E_{j}} \leq c_{22} \inf _{w \in H_{D}^{1}\left(\omega_{z}\right)}\left\|\varepsilon_{\mathcal{T}}\left(w_{h}-w\right)\right\|_{2, \omega_{z}} .
$$

As in the proof of (2.11) and similar to (2.9) we have

$$
\left|a_{j}\right| \leq\left\|h_{\mathcal{E}}^{1 / 2} \partial\left(u_{D}-\mathcal{I} u_{D}\right) / \partial s\right\|_{2, E_{j}}, \quad j=1,2 .
$$

Note also that with $\bigcup \mathcal{E}$ denoting the skeleton of all points on an edge, we have

$$
h_{E_{j}}^{1 / 2}\left\|\partial_{\mathcal{E}} \psi_{j} / \partial s\right\|_{2, \cup \mathcal{E}}+\left\|\nabla_{\mathcal{T}} \psi_{j}\right\|_{2, \omega_{z}} \leq c_{23} .
$$

Combining (4.12)-(4.14) we conclude

$$
\begin{aligned}
& \left\|\partial\left(u_{D}-v_{h}\right) / \partial s\right\|_{2, E_{j}} \\
& \quad \leq\left\|\partial w_{h} / \partial s\right\|_{2, E_{j}}+\left\|\partial\left(u_{D}-\mathcal{I} u_{D}\right) / \partial s\right\|_{2, E_{j}}+c_{23}\left(\left|a_{1}\right| / h_{E_{1}}^{1 / 2}+\left|a_{2}\right| / h_{E_{2}}^{1 / 2}\right) \\
& \quad \leq c_{22} h_{E_{j}}^{-1 / 2} \inf _{w=\mathcal{I} u_{D} \text { on } \Gamma_{D}}\left\|\varepsilon_{\mathcal{T}}\left(v_{h}-w-a_{1} \psi_{1} e_{2}-a_{2} \psi_{2} e_{2}\right)\right\|_{2, \omega_{z}} \\
& \quad+c_{24}\left\|\partial\left(u_{D}-\mathcal{I} u_{D}\right) / \partial s\right\|_{2, \Gamma_{D} \cap \partial \omega_{z}} \\
& \leq c_{22} h_{E_{j}}^{-1 / 2} \inf _{w=\mathcal{I} u_{D} \text { on } \Gamma_{D}}\left\|\varepsilon_{\mathcal{T}}\left(v_{h}-w\right)\right\|_{2, \omega_{z}}+c_{25}\left\|\partial\left(u_{D}-\mathcal{I} u_{D}\right) / \partial s\right\|_{2, \Gamma_{D} \cap \partial \omega_{z}} .
\end{aligned}
$$

To change " $w=\mathcal{I} u_{D}$ on $\Gamma_{D}$ " to " $w=u_{D}$ on $\Gamma_{D}$ " in the infimum, we will prove that

$$
\inf _{w=u_{D}-\mathcal{I} u_{D} \text { on } \Gamma_{D}}\|\varepsilon(w)\|_{2, \omega_{z}} \leq c_{26}\left\|h_{\mathcal{E}}^{1 / 2} \partial\left(u_{D}-\mathcal{I} u_{D}\right) / \partial s\right\|_{2, \Gamma_{D} \cap \partial \omega_{z}} .
$$

Since $u_{D}-\mathcal{I} u_{D}$ vanishes at the endpoints of each edge $E_{j}$, it suffices to prove for an edge $E=E_{j}$ of $T \in \mathcal{T}$ that

$$
\inf _{w=u_{D}-\mathcal{I} u_{D} \text { on } E}\|\varepsilon(w)\|_{2, T} \leq c_{26} h_{E}^{1 / 2}\left\|\partial\left(u_{D}-\mathcal{I} u_{D}\right) / \partial s\right\|_{2, E} .
$$

Several explicit constructions of sufficient $w$ are possible. For instance, let $w \in$ $H^{1}(T)^{2}$ be the harmonic extension of the boundary values $w=u_{D}-\mathcal{I} u_{D}$ on $E$ and $w=0$ on $\partial T \backslash E$. Then,

$$
\|\nabla w\|_{2, T} \leq c_{27}\|w\|_{H^{1 / 2}(\partial T)} .
$$

From a characterisation of the trace space $H^{1 / 2}(\partial T)$ by interpolation of $H^{1}(\partial T)$ and $L^{2}(\partial T)$ we deduce

$$
\|w\|_{H^{1 / 2}(\partial T)} \leq c_{28}\|w\|_{1,2, \partial T}^{1 / 2}\|w\|_{2, \partial T}^{1 / 2}=c_{28}\|w\|_{1,2, E}^{1 / 2}\|w\|_{2, E}^{1 / 2} .
$$


With Young's inequality we infer from (4.18)-(4.19) that

$$
\|\nabla w\|_{2, T} \leq c_{29}\left(h_{E}^{1 / 2}\|\partial w / \partial s\|_{2, E}+h_{E}^{-1 / 2}\|w\|_{2, E}\right) .
$$

A transformation argument shows that the constant $c_{29}$ is $h_{E}$-independent and only depends on the shape of $T$. From (4.20) and a standard estimate of $w=u_{D}-\mathcal{I} u_{D}$, we deduce 4.17) and then (4.16).

The remaining terms such as $h_{E}^{1 / 2}\left\|\left[\partial v_{h} / \partial s\right]\right\|_{2, E}$ for an inner edge in $\omega_{z}$ can be treated similarly utilising an estimate for $h_{E}^{1 / 2}\left\|\left[\partial w_{h} / \partial s\right]\right\|_{2, E}$ as in 4.12). We omit the details.

Lemma 4.6. Let $\omega_{E}:=\bigcup\{T \in \mathcal{T}: E \subset T\}$ denote the neighbourhood $E \in \mathcal{E}$ with $E \not \subset \Gamma_{D}$. Then, there exist $h_{E}$-independent constants $c_{30}, c_{31}, c_{32}, c_{33}$ such that

$$
h_{E}^{1 / 2}\left\|\left[\sigma_{h} n_{E}\right]\right\|_{2, E} \leq c_{30} \inf _{\tau \in H\left(\operatorname{div} ; \omega_{E}\right)^{2}}\left(\left\|\sigma_{h}-\tau\right\|_{2, \omega_{E}}+h_{E}\left\|\operatorname{div}_{\mathcal{T}}\left(\sigma_{h}-\tau\right)\right\|_{2, \omega_{E}}\right)
$$

if $E$ is an inner edge. If $E \subset \bar{\Gamma}_{N}$ and $\omega_{E}=T_{E}$ for some $T_{E} \in \mathcal{T}$, there holds, for each $g_{E} \in P_{k}(E)$,

$$
h_{E}^{1 / 2}\left\|\sigma_{h} n_{E}-g_{E}\right\|_{2, E} \leq c_{31} \inf _{\tau n=g_{E}}\left(\left\|\sigma_{h}-\tau\right\|_{2, T_{E}}+h_{E}\left\|\operatorname{div}_{\mathcal{T}}\left(\sigma_{h}-\tau\right)\right\|_{2, T_{E}}\right),
$$

$$
\begin{aligned}
h_{E}^{1 / 2}\left\|\sigma_{h} n_{E}-g\right\|_{2, E} & \leq h_{E}^{1 / 2}\left\|g_{E}-g\right\|_{2, E} \\
& +c_{32} \inf _{\tau n=g}\left(\left\|\sigma_{h}-\tau\right\|_{2, T_{E}}+c_{33} h_{E}\left\|\operatorname{div}_{\mathcal{T}}\left(\sigma_{h}-\tau\right)\right\|_{2, T_{E}}\right),
\end{aligned}
$$

where in the infima in (4.22) and (4.23), " $\tau n=g_{E}$ " and " $\tau n=g$ " stand for all functions $\tau \in H\left(\operatorname{div} ; \omega_{E}\right)^{2}$ with $\tau n=g_{E}$ and $\tau n=g$ on $E$, respectively.

Proof. The proof follows $\mathrm{V1}$ and considers the $\mathcal{T}$-piecewise quadratic bubble function $b_{E}$ for the edge $E \subset \partial T ; b_{E}$ vanishes on $\partial T \backslash E$ and is normalized by $\max b_{E}=1$. The norms $\|\cdot\|_{2, E}$ and $\left\|b_{E}^{1 / 2} \cdot\right\|_{2, E}$ are equivalent, with equivalent constant $c_{34}$, on the finite dimensional space which $\left[\sigma_{h} n_{E}\right]$ belongs to. Let $E \in \mathcal{E}$ be an inner edge, $E=T_{1} \cap T_{2}$ for some $T_{1}, T_{2} \in \mathcal{T}$. Then using the extension operator $P: C(E) \rightarrow C\left(T_{1} \cup T_{2}\right)$ of $[\mathrm{V} 1$ and

$$
\int_{\omega_{E}}\left\{\nabla\left(b_{E} P\left(\left[\sigma_{h} n_{E}\right]\right)\right): \tau+b_{E} P\left(\left[\sigma_{h} n_{E}\right]\right) \cdot \operatorname{div} \tau\right\} d x=0,
$$

(owing to integration by parts) we infer

$$
\begin{aligned}
\left\|\left[\sigma_{h} n_{E}\right]\right\|_{2, E}^{2} & \leq c_{34} \int_{E} b_{E} P\left(\left[\sigma_{h} n_{E}\right]\right) \cdot\left[\sigma_{h} n_{E}\right] d s \\
& =c_{34} \int_{\omega_{E}}\left\{\nabla\left(b_{E} P\left(\left[\sigma_{h} n_{E}\right]\right)\right)\left(\sigma_{h}-\tau\right)+b_{E} P\left(\left[\sigma_{h} n_{E}\right]\right) \operatorname{div}_{\mathcal{T}}\left(\sigma_{h}-\tau\right)\right\} d x .
\end{aligned}
$$

Cauchy's inequality, the inverse estimate

$$
\left\|\nabla\left(b_{E} P\left(\left[\sigma_{h} n_{E}\right]\right)\right)\right\|_{2, \omega_{E}} \leq c_{35} h_{E}^{-1}\left\|b_{E} P\left(\left[\sigma_{h} n_{E}\right]\right)\right\|_{2, \omega_{E}},
$$

and

$$
\left\|b_{E} P\left(\left[\sigma_{h} n_{E}\right]\right)\right\|_{2, \omega_{E}} \leq c_{36} h_{E}^{1 / 2}\left\|\left[\sigma_{h} n_{E}\right]\right\|_{2, E}
$$


lead to

$$
h_{E}^{1 / 2}\left\|\left[\sigma_{h} n_{E}\right]\right\|_{2, E} \leq c_{34} c_{36}\left\{c_{35}\left\|\sigma_{h}-\tau\right\|_{2, \omega_{E}}+h_{E}\left\|\operatorname{div}_{\mathcal{T}}\left(\sigma_{h}-\tau\right)\right\|_{2, \omega_{E}}\right\} .
$$

The proof of (4.21) is finished. The same arguments prove (4.22) for an edge on the boundary $\Gamma_{N}$ as well by straightforward modifications. We omit the details which lead to (4.22) and mention in the proof of (4.23) only that instead of (4.25) we now study

$$
\begin{aligned}
& \text { (4.27) }\left\|\sigma_{h} n_{E}-g_{E}\right\|_{2, E}^{2} \leq c_{34} \int_{E} b_{E} P\left(\sigma_{h} n_{E}-g_{E}\right) \cdot\left(\sigma_{h} n_{E}-g_{E}\right) d s \\
& \leq c_{34} \int_{E} b_{E} P\left(\sigma_{h} n_{E}-g_{E}\right) \cdot\left(\sigma_{h}-\tau\right) n_{E} d s+c_{34} \int_{E} b_{E} P\left(\sigma_{h} n_{E}-g_{E}\right) \cdot\left(g-g_{E}\right) d s
\end{aligned}
$$

and integrate by parts only in the second to last term.

Proof of Theorem 4.1. We prove (4.3) by combining the estimates of Lemma 4.5 and 4.6. One needs to notice carefully that the conditions on the mesh allow, for any edge $E$ even on the boundary, the choice of some endpoint $z \in \mathcal{N}$ such that $E$ is involved in the patch-oriented estimates.

Notice that $v=u$ and $\tau=\sigma$ can be included and that $\mathcal{A}$ is Lipschitz continuous. To prove (4.4) it remains to observe ( $u$ is divergence free)

$$
\left\|\operatorname{div} u_{h}\right\|_{2, T} \leq\left\|\varepsilon_{\mathcal{T}}\left(u-u_{h}\right)\right\|_{2, T}
$$

and estimate the term div $\sigma_{h}+f_{T}$ following [V1]. To do so, consider the nonnegative cubic bubble function $b_{T}$ on $T \in \mathcal{T}$ with $\max b_{T}=1$. Then, the norms $\|\cdot\|_{2, T}$ and $\left\|b_{T}^{1 / 2} \cdot\right\|_{2, T}$ are equivalent on the finite dimensional space to which $\operatorname{div} \sigma_{h}+f_{T}$ belongs, and so

$$
\begin{aligned}
c_{37}^{-1} \| & \operatorname{div} \sigma_{h}+f_{T} \|_{2, T}^{2} \\
& \leq \int_{T} b_{T}\left(\operatorname{div} \sigma_{h}+f_{T}\right) \cdot\left(\operatorname{div}\left(\sigma_{h}-\sigma\right)-f+f_{T}\right) d x \\
& \leq \int_{T} \nabla\left(b_{T}\left(\operatorname{div} \sigma_{h}+f_{T}\right)\right):\left(\sigma_{h}-\sigma\right) d x+\left\|\operatorname{div} \sigma_{h}+f_{T}\right\|_{2, T}\left\|f-f_{T}\right\|_{2, T} \\
& \leq\left\|\nabla\left(b_{T}\left(\operatorname{div} \sigma_{h}+f_{T}\right)\right)\right\|_{2, T}\left\|\sigma-\sigma_{h}\right\|_{2, T}+\left\|\operatorname{div} \sigma_{h}+f_{T}\right\|_{2, T}\left\|f-f_{T}\right\|_{2, T} .
\end{aligned}
$$

Utilising the inverse estimate $\left\|\nabla\left(b_{T}\left(\operatorname{div} \sigma_{h}+f_{T}\right)\right)\right\|_{2, T} \leq c_{38} h_{T}^{-1}\left\|\operatorname{div} \sigma_{h}+f_{T}\right\|_{2, T}$, we obtain

$$
h_{T}\left\|\operatorname{div} \sigma_{h}+f_{T}\right\|_{2, T} \leq c_{37} c_{38}\left\|\sigma-\sigma_{h}\right\|_{2, T}+c_{37} h_{T}\left\|f-f_{T}\right\|_{2, T} .
$$

\section{Averaging techniques FOR A POSTERIORI ERROR CONTROL}

The ZZ-estimator [ZZ] and estimators often based on gradient recovery techniques can be justified on arbitrary shape-regular meshes by the refined estimate of the previous sections. The first result shows the reliability of low order conforming schemes; below we discuss estimators for the lowest order conformingnonconforming finite element scheme (1.8)-(1.9) .

Let $(u, p) \in H^{1}(\Omega)^{2} \times L^{2}(\Omega)$ solve (1.1)-(1.3) and let $\left(u_{h}, p_{h}\right) \in \mathcal{W}$ with $k=1$ satisfy (1.6)-(1.7). As in Stokes' problem suppose that $\mathcal{A}$ maps deviatoric strains onto deviatoric ones, i.e., $\operatorname{tr} \mathcal{A}(E)=0$ for all $E \in \mathbb{R}_{\text {sym }}^{2 \times 2}$ with $\operatorname{tr} E=0$. 
Let $\mathcal{N}^{*}$ denote the set of all $z \in \mathcal{N}$ which are either free nodes or belong to two aligned edges on $\Gamma_{D}$. If $z \in \mathcal{N}^{*} \cap \Gamma_{D}$ such that $\Gamma_{D} \cap \partial \omega_{z}=E_{1} \cup E_{2}=: \gamma_{z}$ for two aligned distinct edges $E_{1}, E_{2} \in \mathcal{E}$ and such that there exists a third edge $E_{3} \in \mathcal{E} \backslash\left\{E_{1}, E_{2}\right\}$ through $z$ and parallel to the $x_{1}$-axis, then for some interior node $\zeta \in \mathcal{N}$ with $E_{3}=\operatorname{conv}\{z, \zeta\} \subset \mathbb{R} \times\left\{z_{2}\right\}$ (e.g., $\zeta=\zeta(z)$ as in Theorem 2.1) we define $\Omega_{z}:=\omega_{z} \cup \omega_{\zeta}$. In all remaining cases of $z \in \mathcal{N}^{*}$ we define $\Omega_{z}:=\omega_{z}$ and $\gamma_{z}:=\emptyset$.

Theorem 5.1 implies the estimate (1.13) and the reliability of (1.14).

Theorem 5.1. Let $(u, p) \in H^{1}(\Omega)^{2} \times L^{2}(\Omega)$ solve (1.1)-(1.3) and $\left(u_{h}, p_{h}\right) \in H^{2}(\mathcal{T})^{2}$ $\times L^{2}(\Omega)$ solve (1.6)-(1.7). Suppose that $\Gamma_{D}$ is connected and $u_{D} \in C\left(\Gamma_{D}\right) \cap H^{1}\left(\Gamma_{D}\right)$ is piecewise $H^{2}\left(\Gamma_{D}\right)$ in the sense that $\left.u_{D}\right|_{\gamma_{z}} \in H^{2}\left(\gamma_{z}\right)^{2}$ for $z \in \mathcal{N}^{*} \cap \Gamma_{D}$.

Then, there exists an $h$-independent constant $c_{39}>0$ that depends on the shape of the elements and the patches $\left(\omega_{z}: z \in \mathcal{K}\right)$ only such that

$$
\begin{aligned}
& \leq c_{39}\left(\sum _ { z \in \mathcal { N } ^ { * } } \left(\min _{\tau_{z} \in \mathcal{S}^{1}\left(\left.\mathcal{T}\right|_{\omega_{z}}\right)^{2 \times 2}}\left(\left\|\sigma_{h}-\tau_{z}\right\|_{L^{2}\left(\Omega_{z}\right)}^{2}+\left\|h_{\mathcal{E}}^{1 / 2}\left(g-\tau_{z} n\right)\right\|_{L^{2}\left(\Gamma_{N} \cap \partial \omega_{z}\right)}^{2}\right)\right.\right. \\
& \left.\left.+\left\|h_{\mathcal{E}}^{3 / 2} \partial_{\mathcal{E}}^{2} u_{D} / \partial s^{2}\right\|_{L^{2}\left(\Gamma_{D} \cap \partial \omega_{z}\right)}^{2}\right)\right)^{1 / 2}+c_{7}\left(\sum_{z \in \mathcal{K}} \min _{f_{z} \in \mathbb{R}^{2}}\left\|h_{\mathcal{T}}\left(f-f_{z}\right)\right\|_{L^{2}\left(\Omega_{z}\right)}^{2}\right)^{1 / 2} .
\end{aligned}
$$

Moreover, suppose $g \in C\left(\Gamma_{N}\right)^{2}$ is $\mathcal{E}$-piecewise in $H^{1}\left(\Gamma_{N}\right)^{2}$, i.e., $\left.g\right|_{E} \in H^{1}(E)^{2}$ for $E \in \mathcal{E}$ with $E \subset \bar{\Gamma}_{N}$. Assume that $\sigma_{h}^{*} \in \mathcal{S}^{1}(\mathcal{T})^{2 \times 2}$ satisfies $g(z)=\sigma_{h}^{*}(z) n_{E}(z)$ for each endpoint $z$ of an edge $E$ on $\bar{\Gamma}_{N}$. Then,

$$
\begin{aligned}
& \left\|\varepsilon_{\mathcal{T}}\left(u-u_{h}\right)\right\|_{L^{2}(\Omega)}+\left\|p-p_{h}\right\|_{L^{2}(\Omega)} \\
& \quad \leq c_{39}\left(\left\|\sigma_{h}-\sigma_{h}^{*}\right\|_{L^{2}(\Omega)}+\left\|h_{\mathcal{E}}^{3 / 2} \partial_{\mathcal{E}}^{2} u_{D} / \partial s^{2}\right\|_{L^{2}\left(\Gamma_{D}\right)}+\left\|h_{\mathcal{E}}^{3 / 2} \partial_{\mathcal{E}} g / \partial s\right\|_{L^{2}\left(\Gamma_{D}\right)}\right) \\
& \quad+c_{7}\left(\sum_{z \in \mathcal{K}} \min _{f_{z} \in \mathbb{R}^{2}}\left\|h_{\mathcal{T}}\left(f-f_{z}\right)\right\|_{L^{2}\left(\Omega_{z}\right)}^{2}\right)^{1 / 2}
\end{aligned}
$$

Remarks 5.1. (i) If $g$ is $\mathcal{E}$-piecewise in $H^{2}\left(\Gamma_{N}\right)^{2}$, the perturbation term $\left\|h_{\mathcal{E}}^{3 / 2} \partial g / \partial s\right\|_{2}$ in (5.2) can be improved to $\left\|h_{\mathcal{E}}^{5 / 2} \partial_{\mathcal{E}}^{2} g / \partial s^{2}\right\|_{2}$.

(ii) The discrete Neumann boundary conditions on the nonsymmetric $\sigma_{h}^{*}$ can be satisfied exactly even at corner points (with two different normals); see (6.2.i)(6.2.ii) below for details.

(iii) The choice of the remaining degrees of freedom in $\sigma_{h}^{*}$ is arbitrary: any averaging scheme is reliable. The efficiency of the averaging process is a different topic and has to be checked separately.

(iv) It is interesting to notice that the higher order terms in the reliability estimate depend on the smoothness of the data while the the higher order terms in the efficiency estimate depend on the smoothness of the exact solution. 
Lemma 5.1. For any $z \in \mathcal{N}^{*}$ there exists an $h$-independent constant $c_{40}>0$ such that, for all $v_{h} \in \mathcal{W}$ (for $k=1$ ),

$$
\begin{aligned}
& \text { 5.3) }\left(\sum_{E \sqsubset \Omega_{z}} h_{E}\left\|\left[\partial v_{h} / \partial s\right]\right\|_{L^{2}(E)}^{2}\right)^{1 / 2} \\
& \leq c_{40}\left(\left\|h_{\mathcal{E}}^{3 / 2} \partial^{2}\left(u_{D}-\mathcal{I} u_{D}\right) / \partial s^{2}\right\|_{L^{2}\left(\Gamma_{D} \cap \partial \omega_{z}\right)}+\min _{V_{h}, \tau_{h}}\left\|\varepsilon\left(v_{h}-V_{h}\right)-\tau_{h}\right\|_{L^{2}\left(\Omega_{z}\right)}\right) .
\end{aligned}
$$

In the minimum of (5.3), $V_{h}$ is arbitrary in $\mathcal{S}_{D}^{1}\left(\left.\mathcal{T}\right|_{\Omega_{z}}\right):=\left\{V \in C\left(\Omega_{z}\right)^{2} \cap \mathcal{S}^{1}\left(\left.\mathcal{T}\right|_{\Omega_{z}}\right)^{2}\right.$ : $V=0$ on $\left.\gamma_{z}\right\}$ and $\tau_{h}$ is arbitrary in $\mathcal{S}^{1}\left(\left.\mathcal{T}\right|_{\Omega_{z}}\right)^{2 \times 2}=\left\{\left(\tau_{j k}\right) \in C\left(\Omega_{z}\right)^{2 \times 2}: \forall T \in\right.$ $\mathcal{T},\left.\tau_{j k}\right|_{T} \in P_{1}(T)$ provided $\left.T \subset \overline{\Omega_{z}}\right\}$.

Proof. In the first step of the proof we consider $u_{D}=0$ and show that the right-hand side of (5.3) is a norm on the space $\mathcal{W}_{z} / \mathcal{C}_{z}$ from (4.10). To check definiteness, suppose that $v_{h} \in \mathcal{W}_{z}, V_{h} \in \mathcal{S}_{D}^{1}\left(\left.\mathcal{T}\right|_{\Omega_{z}}\right)$, and $\tau_{h} \in \mathcal{S}^{1}\left(\left.\mathcal{T}\right|_{\Omega_{z}}\right)^{2 \times 2}$ satisfies $\tau_{h}=\varepsilon_{\mathcal{T}}\left(v_{v}-V_{h}\right)$ and so the $\mathcal{T}$-piecewise constant function $\varepsilon_{\mathcal{T}}\left(v_{v}-V_{h}\right)$ is continuous, where it is constant on $\Omega_{z}$. Thus we can find an affine mapping $A x+b$ such that $r(x):=v_{h}(x)-V_{h}(x)-A x-b$ satisfies $\varepsilon_{\mathcal{T}}(r)=0$ on $\Omega_{z}$, i.e., $\left.r\right|_{T} \in \mathcal{R} \mathcal{M}(T)$ for all $T \in \mathcal{T}$ with $T \subset \overline{\Omega_{z}}$. The compatibility conditions on the edges for $v_{h}$ imply the same for $r$ and so Lemma 4.4 shows $r \in \mathcal{R} \mathcal{M}\left(\omega_{z}\right)$ if $z$ is an interior node or if no edge $E \sqsubset \omega_{z}$ is parallel to the $x_{1}$-axis. In the case that $z \in \Gamma_{D}$ we have $\Gamma_{D} \cap \partial \omega_{z}=E_{1} \cup E_{2}$ for two aligned distinct edges $E_{1}, E_{2} \in \mathcal{E}$. If they are parallel to the $x_{1}$-axis we have $r \in \mathcal{R} \mathcal{M}\left(\omega_{z}\right)$ from Lemma 4.4 If there exists another edge $E_{3}=\operatorname{conv}\{z, \zeta\} \subset \mathbb{R} \times\left\{z_{2}\right\}$ we have $r \in \mathcal{R} \mathcal{M}\left(\omega_{\zeta}\right)$. Since $r$ is the same rigid body motion on both elements joining $E_{3}$ we find $r \in \mathcal{R} \mathcal{M}\left(\Omega_{z}\right)$ using Lemma 4.2. The interior jumps in the left-hand side of (5.3) vanish in any case. The boundary contributions vanish as well since the affine function $v_{h}-V_{h}$ vanishes at the midpoint $m_{j}$ of $E_{j}$ for $j=1,2$ and so on the straight line through $\gamma_{z}$.

We have seen that the left-hand side of (5.3) vanishes if the right-hand side does. A compactness and a scaling argument concludes the proof of the lemma if $u_{D}=0$.

In the second part of the proof, we have $z \in \Gamma_{D} \cap E_{1} \cap E_{2}$ for two aligned distinct edges $E_{j}=\operatorname{conv}\left\{z, \zeta_{j}\right\} \subset \Gamma_{D}, j=1,2$, and allow $u_{D} \neq 0$. Extend $\mathcal{I} u_{D}$ (prescribing remaining nodal values) to $\mathcal{I} u_{D} \in \mathcal{S}^{1}(\mathcal{T})^{2}$ and define the affine functions

$$
\begin{gathered}
a_{1}(x):=\frac{\left(x-\zeta_{1}\right) \cdot\left(\zeta_{2}-\zeta_{1}\right)}{\left|\zeta_{2}-\zeta_{1}\right|^{2}} e_{1} \cdot u_{D}\left(\zeta_{2}\right)+\frac{\left(\zeta_{2}-x\right) \cdot\left(\zeta_{2}-\zeta_{1}\right)}{\left|\zeta_{2}-\zeta_{1}\right|^{2}} e_{1} \cdot u_{D}\left(\zeta_{1}\right) \\
a_{2}(x):=\frac{\left(x-m_{1}\right) \cdot\left(m_{2}-m_{1}\right)}{h_{E_{2}}\left|m_{2}-m_{1}\right|^{2}} \int_{E_{2}} e_{2} \cdot u_{D} d s+\frac{\left(m_{2}-x\right) \cdot\left(m_{2}-m_{1}\right)}{h_{E_{1}}\left|m_{2}-m_{1}\right|^{2}} \int_{E_{1}} e_{2} \cdot u_{D} d s
\end{gathered}
$$

The discrete function

$$
w_{h}:=v_{h}-\left(e_{1} \cdot \mathcal{I} u_{D}\right) e_{1}-a_{2} e_{2}
$$

satisfies the compatibility conditions for homogeneous Dirichlet data considered in the first step of this proof. Hence, we obtain in particular, for $j=1,2$,

$$
h_{E_{j}}^{1 / 2}\left\|\partial w_{h} / \partial s\right\|_{2, E_{j}} \leq c_{22} \min _{V_{h}, \tau_{h}}\left\|\varepsilon\left(w_{h}-V_{h}\right)-\tau_{h}\right\|_{2, \Omega_{z}} .
$$

Notice that $\tau_{h}=\varepsilon\left(a_{1} e_{1}+a_{2} e_{2}\right)$ is constant and so allowed in the minimum in (5.7). Hence we may replace $w_{h}$ in (5.7) by $\tilde{w}_{h}:=w_{h}+a_{1} e_{1}+a_{2} e_{2}-V_{h}$ where $V_{h}$ is such that $\left(a_{1}-e_{1} \cdot \mathcal{I} u_{D}\right) e_{1}-V_{h}$ vanishes at all nodes different from $z\left(a_{1}-e_{1} \cdot \mathcal{I} u_{D}\right.$ has zeros $\zeta_{1}, \zeta_{2}$ by construction). This shows $\tilde{w}_{h}=v_{h}+\left(a_{1}(z)-e_{1} \cdot u_{D}(z)\right) \varphi_{z} e_{1}$ with $\varphi_{z}$ being the nodal basis function at $z$. From $\left\|\nabla \varphi_{z}\right\|_{2} \leq c_{41}$ and a triangle 
inequality we deduce

$$
\begin{aligned}
\min _{V_{h}, \tau_{h}}\left\|\varepsilon\left(w_{h}-V_{h}\right)-\tau_{h}\right\|_{2, \Omega_{z}} \\
\quad \leq \min _{V_{h}, \tau_{h}}\left\|\varepsilon\left(v_{h}-V_{h}\right)-\tau_{h}\right\|_{2, \Omega_{z}}+c_{41}\left|a_{1}(z)-e_{1} \cdot u_{D}(z)\right| .
\end{aligned}
$$

As in (4.15), we infer

$$
\begin{aligned}
h_{E_{j}}^{1 / 2} \| & \partial\left(u_{D}-v_{h}\right) / \partial s\left\|_{2, E_{j}} \leq h_{E_{j}}^{1 / 2}\right\| \partial w_{h} / \partial s \|_{2, E_{j}} \\
& +h_{E_{j}}^{1 / 2}\left\|\partial e_{1} \cdot\left(u_{D}-\mathcal{I} u_{D}\right) / \partial s\right\|_{2, E_{j}}+h_{E_{j}}^{1 / 2}\left\|\partial\left(a_{2}-e_{2} \cdot u_{D}\right) / \partial s\right\|_{2, E_{j}} .
\end{aligned}
$$

Standard arguments in one dimension show

$$
\begin{aligned}
& \left|a_{1}(z)-e_{1} \cdot u_{D}(z)\right|+h_{E_{j}}^{1 / 2}\left\|\partial e_{1} \cdot\left(u_{D}-\mathcal{I} u_{D}\right) / \partial s\right\|_{2, E_{j}} \\
& +h_{E_{j}}^{1 / 2}\left\|\partial\left(a_{2}-e_{2} \cdot u_{D}\right) / \partial s\right\|_{2, E_{j}} \leq c_{42}\left\|h_{\mathcal{E}}^{3 / 2} \partial^{2}\left(u_{D}-\mathcal{I} u_{D}\right) / \partial s^{2}\right\|_{2, E_{1} \cup E_{2}} .
\end{aligned}
$$

Combining (5.7)-(5.10) we conclude that (5.3) holds.

Proof of Theorem [5.1. Some terms in Theorem 2.1 simplify because $u_{h}$ is $\mathcal{T}$-piecewise affine. For instance, $\operatorname{div}_{\mathcal{T}} \sigma_{h}=0$ and $\operatorname{div}_{\mathcal{T}} u_{h}=0$. The term $\inf _{v} \| \nabla_{\mathcal{T}}\left(u_{h}-\right.$ $v) \|_{2}$ can be bounded as in (2.7) of Remark 2.1 The remaining edge contributions are estimated with Lemmas 4.6 and 5.1 (with $V_{h}=0$ ) in a manner similar to the proof of Theorem 4.1 In this way, we obtain

$$
\begin{aligned}
& \text { (5.11) } \quad\left\|\varepsilon_{\mathcal{T}}\left(u-u_{h}\right)\right\|_{2}^{2}+\left\|p-p_{h}\right\|_{2}^{2} \leq c_{7}^{2} \sum_{z \in \mathcal{K}} \min _{f_{z} \in \mathbb{R}^{2}}\left\|h_{\mathcal{T}}\left(f-f_{z}\right)\right\|_{2, \Omega_{z}}^{2} \\
& \quad+c_{43} \sum_{z \in \mathcal{N}^{*}}\left(\operatorname { m i n } _ { \tau _ { z } , \tilde { \tau } _ { z } \in \mathcal { S } ^ { 1 } ( \mathcal { T } | _ { \omega _ { z } } ) ^ { 2 \times 2 } } \left(\left\|\sigma_{h}-\tau_{z}\right\|_{2, \Omega_{z}}^{2}+h_{z}^{2}\left\|\operatorname{div}_{\mathcal{T}}\left(\sigma_{h}-\tau_{z}\right)\right\|_{2, \Omega_{z}}^{2}\right.\right. \\
& \left.+\left\|\varepsilon_{\mathcal{T}}\left(u_{h}\right)-\tilde{\tau}_{z}\right\|_{2, \Omega_{z}}^{2}+\left\|h_{\mathcal{E}}^{1 / 2}\left(g-\tau_{z} n\right)\right\|_{2, \Gamma_{N} \cap \partial \omega_{z}}^{2}+\left\|h_{\mathcal{E}}^{3 / 2} \partial_{\mathcal{E}}^{2} u_{D} / \partial s^{2}\right\|_{2, \Gamma_{D} \cap \partial \omega_{z}}^{2}\right) .
\end{aligned}
$$

An inverse estimate shows for one summand in (5.11) that

$$
h_{z}\left\|\operatorname{div}_{\mathcal{T}}\left(\sigma_{h}-\tau_{z}\right)\right\|_{2, \Omega_{z}} \leq\left\|\sigma_{h}-\tau_{z}\right\|_{2, \Omega_{z}} .
$$

The equivalence of norms and a scaling argument for the $h_{z}$-independent constant $c_{45}>0$ shows for $\mathcal{T}$-piecewise constants $\mathcal{L}_{0}\left(\left.\mathcal{T}\right|_{\Omega_{z}}\right)$ and the continuous $\mathcal{T}$-piecewise affine functions $\mathcal{S}^{1}\left(\left.\mathcal{T}\right|_{\Omega_{z}}\right)$ that, for all $\alpha \in \mathcal{L}_{0}\left(\left.\mathcal{T}\right|_{\Omega_{z}}\right)^{2 \times 2}$,

$$
c_{45} \min _{\gamma \in \mathbb{R}_{s y m}^{2 \times 2}}\|\alpha-\gamma\|_{2, \Omega_{z}} \leq \min _{\beta \in \mathcal{S}^{1}\left(\left.\mathcal{T}\right|_{\Omega_{z}}\right)_{\text {sym }}^{2 \times 2}}\|\alpha-\beta\|_{2, \Omega_{z}} \leq \min _{\gamma \in \mathbb{R}_{s y m}^{2 \times 2}}\|\alpha-\gamma\|_{2, \Omega_{z}} .
$$

A Cauchy inequality and (1.4) reveal that for all $\alpha, \beta \in \mathbb{R}_{\text {sym }}^{2 \times 2}$,

$$
c_{1}|\alpha-\beta| \leq|\mathcal{A}(\alpha)-\mathcal{A}(\beta)| \leq c_{2}|\alpha-\beta| .
$$

Owing to monotonicity arguments, the mapping $\mathcal{A}$ is a bijection on $\mathbb{R}_{\text {sym }}^{2 \times 2}$ and so we deduce from (5.13)-(5.14) for the piecewise constant $\varepsilon_{\mathcal{T}}\left(u_{h}\right)$ that

$$
\begin{gathered}
c_{1} \min _{\tilde{\tau}_{z} \in \mathcal{S}^{1}\left(\left.\mathcal{T}\right|_{\Omega_{z}}\right)_{s y m}^{2 \times 2}}\left\|\varepsilon_{\mathcal{T}}\left(u_{h}\right)-\tilde{\tau}_{z}\right\|_{2, \Omega_{z}} \leq c_{1} \min _{\gamma \in \mathbb{R}_{s y m}^{2 \times 2}}\left\|\varepsilon_{\mathcal{T}}\left(u_{h}\right)-\gamma\right\|_{2, \Omega_{z}} \\
\leq \min _{\gamma \in \mathbb{R}_{s y m}^{2 \times 2}}\left\|\mathcal{A}\left(\varepsilon_{\mathcal{T}}\left(u_{h}\right)\right)-\mathcal{A}(\gamma)\right\|_{2, \Omega_{z}}=\min _{\gamma \in \mathbb{R}_{s y m}^{2 \times 2}}\left\|\mathcal{A}\left(\varepsilon_{\mathcal{T}}\left(u_{h}\right)\right)-\gamma\right\|_{2, \Omega_{z}} \\
\leq c_{45}^{-1} \min _{\tau_{h} \in \mathcal{S}^{1}\left(\left.\mathcal{T}\right|_{\Omega_{z}}\right)_{s y m}^{2 \times 2}}\left\|\mathcal{A}\left(\varepsilon_{\mathcal{T}}\left(u_{h}\right)\right)-\tau_{h}\right\|_{2, \Omega_{z}} \\
=c_{45}^{-1} \min _{\tau_{h} \in \mathcal{S}^{1}\left(\left.\mathcal{T}\right|_{\Omega_{z}}\right)_{s y m}^{2 \times 2}}\left\|\operatorname{dev}\left(\sigma_{h}-\tau_{h}\right)\right\|_{2, \Omega_{z}} \leq c_{45}^{-1} \min _{\tau_{h} \in \mathcal{S}^{1}\left(\left.\mathcal{T}\right|_{\Omega_{z}}\right)_{s y m}^{2 \times 2}}\left\|\sigma_{h}-\tau_{h}\right\|_{2, \Omega_{z}} .
\end{gathered}
$$


Here we used $\operatorname{dev} A=A-\operatorname{tr}(A) / 2 I$ and, in the second to last step, that $\mathcal{A}\left(\varepsilon_{\mathcal{T}}\left(u_{h}\right)\right)=$ $\sigma_{h}-p_{h} I$ is pointwise almost everywhere deviatoric $\left(\right.$ since $0=\operatorname{div}_{\mathcal{T}} u=\operatorname{tr} \varepsilon_{\mathcal{T}}\left(u_{h}\right)$ ) and so is the optimal $\tau_{h}$.

A combination of (5.11), (5.12), and (5.15) concludes the proof of Theorem 5.1

\section{NumERICAL EXPERIMENTS}

In this section we report on the numerical performance of the two a posteriori estimates established in this paper (Theorems 2.1 and 5.1) and give two strategies to refine a given mesh automatically.

The proposed estimator in Theorem [5.1 is based on a function $\sigma_{h}^{*} \in \mathcal{S}^{1}(\mathcal{T})^{2 \times 2}$ which satisfies $g(z)=\sigma_{h}^{*}(z) n_{E}(z)$ for each endpoint $z$ of an edge $E$ on $\bar{\Gamma}_{N}$. We define

$$
\sigma_{h}^{*}:=\sum_{z \in \mathcal{N}} \mathcal{I}_{z} \sigma_{h} \varphi_{z}
$$

where $\mathcal{I}: L^{2}(\Omega)^{2 \times 2} \rightarrow \mathcal{S}^{1}(\mathcal{T})^{2 \times 2}$ for $z \in \mathcal{N} \backslash \bar{\Gamma}_{N}$ is, with $f_{\omega_{z}} \sigma_{h} d x$ denoting the integral mean of $\sigma_{h}$ over $\Omega_{z}$,

$$
\mathcal{I}_{z} \sigma_{h}:=f_{\omega_{z}} \sigma_{h} d x
$$

For $z \in \bar{\Gamma}_{N}$ we distinguish between the following cases (i) and (ii) to fulfill the discrete Neumann condition $g(z)=\sigma_{h}^{*}(z) n_{E}$ at $z$.

(6.2.i) In case $z \in E_{1} \cap E_{2}$ for two distinct edges $E_{1}, E_{2} \subset \Gamma_{N}$ with linearly independent outer unit normals $n_{E_{1}}$ and $n_{E_{2}}$ on $E_{1}$ and $E_{2}$, respectively, we choose $\mathcal{I}_{z} \sigma_{h}$ to be the unique solution $\left(\begin{array}{ccc}x_{11} & x_{12} \\ x_{21} & x_{22}\end{array}\right)$ of the linear system

$$
\left(\begin{array}{cccc}
n_{1, E_{1}} & n_{2, E_{1}} & 0 & 0 \\
0 & 0 & n_{1, E_{1}} & n_{2, E_{1}} \\
n_{1, E_{2}} & n_{2, E_{2}} & 0 & 0 \\
0 & 0 & n_{1, E_{2}} & n_{2, E_{2}}
\end{array}\right)\left(\begin{array}{l}
x_{11} \\
x_{12} \\
x_{21} \\
x_{22}
\end{array}\right)=\left(\begin{array}{c}
\left.g_{1}\right|_{E_{1}}(z) \\
g_{2} \mid E_{1}(z) \\
\left.g_{1}\right|_{E_{2}}(z) \\
\left.g_{2}\right|_{E_{2}}(z)
\end{array}\right) .
$$

(6.2.ii) In the remaining cases $z \in E_{1} \cap \Gamma_{D}$ or $z \in E_{1} \cap E_{2}$ with two parallel outer unit normals $n_{E_{1}}, n_{E_{2}}$, we choose $t_{E_{1}}$ to be the unit tangent to $\Omega$ at $z$ that is perpendicular to $n_{E_{1}}$, and let $\mathcal{I}_{z} \sigma_{h}$ be the solution $\left(\begin{array}{ll}x_{11} & x_{12} \\ x_{21} & x_{22}\end{array}\right)$ of the uniquely solvable system

$$
\left(\begin{array}{cccc}
n_{1, E_{1}} & n_{2, E_{1}} & 0 & 0 \\
0 & 0 & n_{1, E_{1}} & n_{2, E_{1}} \\
t_{1, E_{2}} & t_{2, E_{2}} & 0 & 0 \\
0 & 0 & t_{1, E_{2}} & t_{2, E_{2}}
\end{array}\right)\left(\begin{array}{c}
x_{11} \\
x_{12} \\
x_{21} \\
x_{22}
\end{array}\right)=\left(\begin{array}{c}
\left.g_{1}\right|_{E_{1}}(z) \\
\left.g_{2}\right|_{E_{1}}(z) \\
\left(f_{\omega_{z}}\left(\sigma_{h, 11}, \sigma_{h, 12}\right) d x\right) t_{E_{1}} \\
\left(f_{\omega_{z}}\left(\sigma_{h, 21}, \sigma_{h, 22}\right) d x\right) t_{E_{1}}
\end{array}\right)
$$

In the error indicator $\eta_{Z, T}$ this amounts to, for each $T \in \mathcal{T}$,

$$
\eta_{Z, T}:=\left\|\sigma_{h}-\sigma_{h}^{*}\right\|_{L^{2}(T)} .
$$

Since the symmetric formulation with $P_{1}^{2} \times P_{0}$-finite elements is unstable for the conforming and the nonconforming case, we considered the conforming-nonconforming scheme from (1.8)-(1.9).

The implementation is performed on triangles in Matlab in the spirit of $\mathrm{ACF}$ using analytic formulae in the calculation of the stiffness matrix. Since $\mathcal{A}(\alpha)=2 \mu \alpha$ 
in (1.2) is a linear operator in our examples, the linear system of equations can be solved directly. In order to approximate the right-hand side for a given function $g \in L^{2}\left(\Gamma_{N}\right)^{2}$, we compute $\int_{\Gamma_{N}} g v_{h} d s$ via a three-point Gaussian quadrature rule on any edge $E$. The Dirichlet boundary conditions are implemented as in Remark 2.1(viii).

In the comparison of uniform mesh-refinement with adaptive refinement techniques we use the following adaptive Algorithms $\left(A_{R}\right)$ and $\left(A_{Z}\right)$. Both algorithms are different in the error indicators only.

Algorithm 6.1. $\left(A_{R}\right)$ resp. $\left(A_{Z}\right) \quad$ (a) Start with a coarse mesh $\mathcal{T}_{0}, k=0$.

(b) Solve the discrete problem with respect to the actual mesh $\mathcal{T}_{k}$.

(c) Compute $\eta_{T}$ for all $T \in \mathcal{T}_{k}$, where, for $\left(A_{R}\right)$,

$$
\eta_{T}^{2}=\eta_{R, T}^{2}:=h_{T}^{4}\|\nabla f\|_{L^{2}(T)}^{2}+\sum_{E \in \mathcal{E} \wedge E \subset \partial T} h_{E}\left(\left\|\left[\Sigma n_{E}\right]\right\|_{L^{2}(E)}^{2}+\|[2 \mu \partial U / \partial s]\|_{L^{2}(E)}^{2}\right)
$$

and, for $\left(A_{Z}\right)$, with an averaged function $\sigma_{h}^{*}$ of the discrete stress field $\sigma_{h}$ as in (6.1),

$$
\eta_{T}=\eta_{Z, T}:=\left\|\sigma_{h}-\sigma_{h}^{*}\right\|_{L^{2}(T)} .
$$

(d) Compute a given stopping criterion and decide to terminate or to go to (e).

(e) Refine the element $T$ (red refinement) provided,

$$
\frac{1}{2} \max _{T^{\prime} \in \mathcal{T}_{k}} \eta_{T^{\prime}} \leq \eta_{T}
$$

(f) Refine further elements (red-green-blue refinement) to avoid hanging nodes. Define the resulting mesh as the actual mesh $\mathcal{T}_{k+1}$, update $k$ and go to (b).

Remarks 6.1. (i) Details on the so-called red-green-blue refinement strategies can be found in $\mathrm{V2}$.

(ii) Stopping criteria for termination in step (d) can be based on $\eta_{\mathcal{T}}:=$ $\left(\sum_{T \in \mathcal{T}} \eta_{T}^{2}\right)^{1 / 2}$. For instance, we can terminate in $(\mathrm{d})$ if $\eta_{\mathcal{T}_{k}}$ is less then a certain percentage of $\eta_{\mathcal{T}_{0}}$. If $f$ is sufficiently smooth and the mesh is sufficiently fine, $\eta_{Z}$ might be regarded as a very good guess for the exact error.

(iii) Utilising the initial mesh displayed in Figure 2, Algorithm $\left(A_{R}\right)\left(\operatorname{resp} .\left(A_{Z}\right)\right)$ generates a sequence of meshes which satisfy the assumptions of Section 4

Example 6.1. The first numerical example for the Stokes problem is on the Lshaped domain $\Omega:=(-1,1)^{2} \backslash[0,1] \times[-1,0]$ with $f=0$ and $\mathcal{A}(\alpha)=2 \alpha[\mathrm{V} 2]$. The geometries of $\Omega, \Gamma_{D}$ and $\Gamma_{N}$ are depicted in Figure 2 where $\mathcal{T}_{0}$ is shown as well. The boundary values $u_{D}, g$ are taken from the exact solution $(u, p)$ which reads, in polar coordinates for $\alpha=856399 / 1572864 \approx .54448, \omega=3 \pi / 2$,

$$
\begin{aligned}
u(r, \varphi)= & r^{\alpha}\left((1+\alpha)(\sin (\varphi),-\cos (\varphi)) w(\varphi)+(\cos (\varphi), \sin (\varphi)) w_{\varphi}(\varphi)\right), \\
p(r, \varphi)= & -r^{\alpha-1}\left((1+\alpha)^{2} w_{\varphi}(\varphi)+w_{\varphi \varphi \varphi}(\varphi)\right) /(1-\alpha), \\
w(\varphi)= & (\sin ((1+\alpha) \varphi) \cos (\alpha \omega)) /(1+\alpha)-\cos ((1+\alpha) \varphi) \\
& -(\sin ((1-\alpha) \varphi) \cos (\alpha \omega)) /(1-\alpha)+\cos ((1-\alpha) \varphi) .
\end{aligned}
$$

A plot of the mesh $\mathcal{T}_{9}$ generated by Algorithm $\left(A_{R}\right)$ as some magnified detail near the re-entrant corner (zoom of $\left.(-0.1,0.1)^{2}\right)$ is given in Figure 3 and shows a high refinement of the mesh near the singularity at the origin.

The resulting improvement of the convergence is outlined in Figure 4 , where the error $e_{N}:=\left\|2 \varepsilon\left(u-u_{h}\right)-\left(p-p_{h}\right) I\right\|_{L^{2}(\Omega)}$ is plotted versus the number of degrees of 


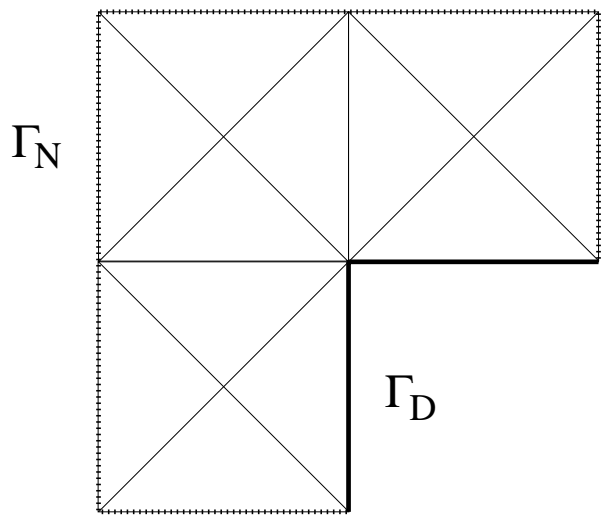

Figure 2. Initial mesh $\mathcal{T}_{0}$ of the domain $\Omega$ and boundary $\Gamma_{D}$, resp. $\Gamma_{N}$ in Example 6.1.

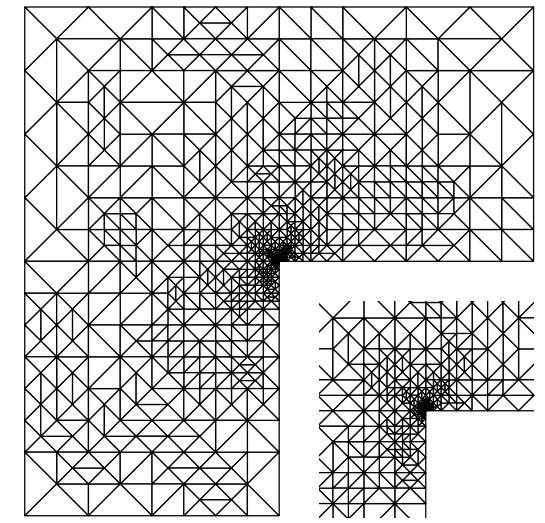

Figure 3 . Mesh $\mathcal{T}_{9}$ and magnified detail at the re-entrant corner in Example 6.1.

freedom $N$ on a $\log / \log$-scale. (A slope $-1 / 2$ in Figures 4 and 5 corresponds to an experimental convergence rate 1 owing to $N \propto h^{-2}$ in two dimensions.) Figure 4 shows the convergence rates for the uniform refinement in comparison with the mentioned adaptive Algorithm $\left(A_{R}\right)$ or $\left(A_{Z}\right)$; eta_R (eta_Z adapted) corresponds to $\eta_{R}$ for a sequence of meshes generated by Algorithm $\left(A_{Z}\right)$. According to the reentrant corner, the uniform refinement yields a convergence rate of approximately 0.544 which coincides with the theoretically expected rate. The adaptive meshrefining Algorithms $\left(A_{Z}\right)$ and $\left(A_{R}\right)$ improve this experimental convergence order to 1 which is expected to be optimal for $\mathcal{V}_{1} \times \mathcal{V}_{2}$-elements.

In Table 1 we displayed the errors and the bounds for different meshes computed with uniform refinements. Here, $N$ is the number of unknowns, $e_{N}$ is the error-norm (evaluated by using a 7-point Gauss quadrature formula of order 6 on each element), and $\eta_{R}$ (resp. $\eta_{Z}$ ) is the computed upper bound of the a posteriori estimate. From Table 1 and Figure 4 we observe that the quotients $\eta_{R} / e_{N}$ (resp. $\eta_{Z} / e_{N}$ ) remain bounded from above in agreement with our theoretical results. Moreover, the quotient of overestimation $\eta_{R} / e_{N}$ is approximately 2.5 for uniform refinements (Table 1) and becomes slightly larger $(\approx 3.3)$ for the adaptive strategies $\left(A_{R}\right)$ and $\left(A_{Z}\right)$. The error estimator $\eta_{Z}$ estimates the error asymptotically exactly for uniform refinements and both adaptive strategies which could result from local symmetries

TABLE 1. Errors $e_{N}$ and error estimates $\eta_{R}, \eta_{Z}$ for uniform meshes of Example 6.1.

\begin{tabular}{|c|c||c|c||c|c|}
\hline$N$ & $e_{N}$ & $\eta_{R}$ & $\eta_{R} / e_{N}$ & $\eta_{Z}$ & $\eta_{Z} / e_{N}$ \\
\hline 45 & 5.1346 & 9.4007 & 1.8308 & 4.8630 & 0.9471 \\
161 & 4.0600 & 8.7255 & 2.1491 & 4.0150 & 0.9889 \\
609 & 2.9155 & 6.8511 & 2.3499 & 2.8915 & 0.9918 \\
2369 & 2.0357 & 4.9026 & 2.4082 & 2.0232 & 0.9939 \\
9345 & 1.4075 & 3.4146 & 2.4260 & 1.4003 & 0.9949 \\
37121 & 0.9690 & 2.3575 & 2.4327 & 0.9646 & 0.9954 \\
147969 & 0.6658 & 1.6219 & 2.4356 & 0.6629 & 0.9956 \\
\hline
\end{tabular}




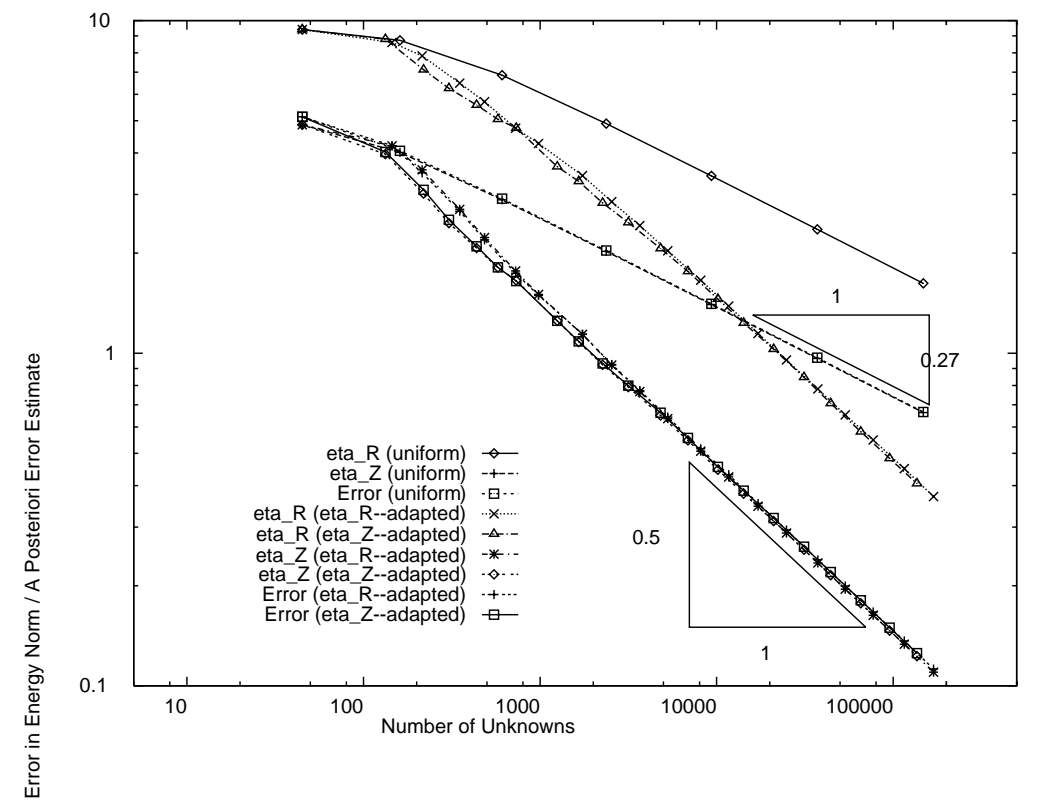

Figure 4. Errors $e_{N}$ vs $N$ for uniform and adaptive meshes of Example 6.1]

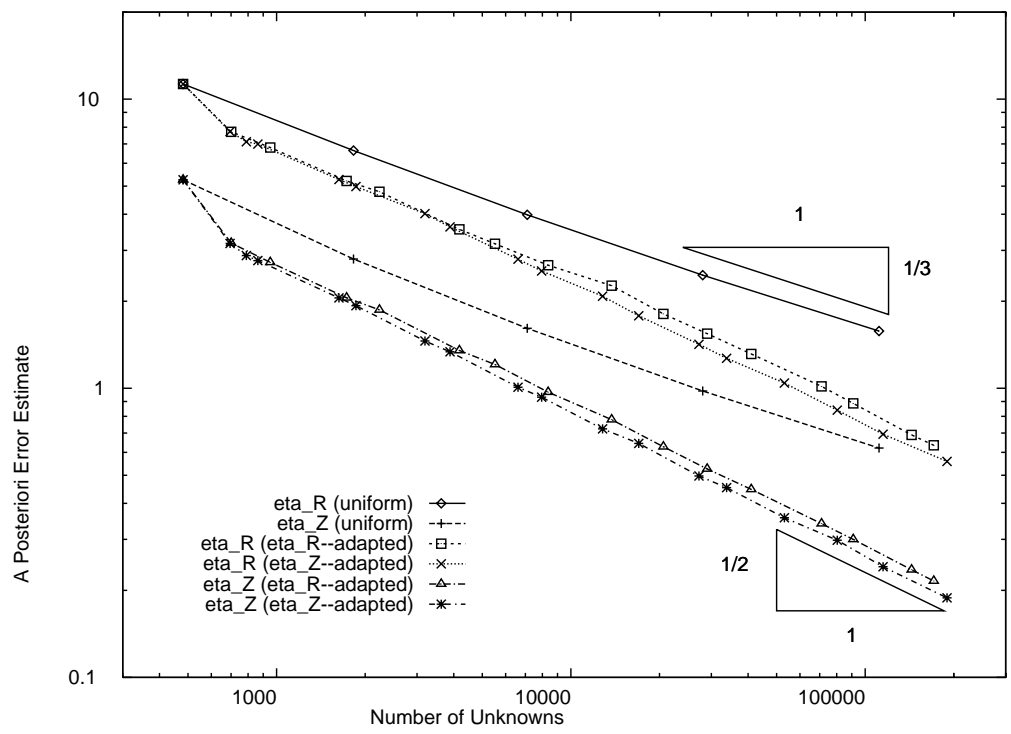

FiguRE 5. A posteriori error indicator $\eta_{R}$ and $\eta_{Z}$ vs $N$ for uniform and adaptive meshes of Example 6.2

in the mesh and superconvergence. Preasymptotically we obtain by Algorithm $\left(A_{Z}\right)$ meshes with slightly smaller errors $e_{N}$ and quantities $\eta_{R}$ and $\eta_{Z}$. This numerical example supports the assertion that the estimates are reliable and efficient. 


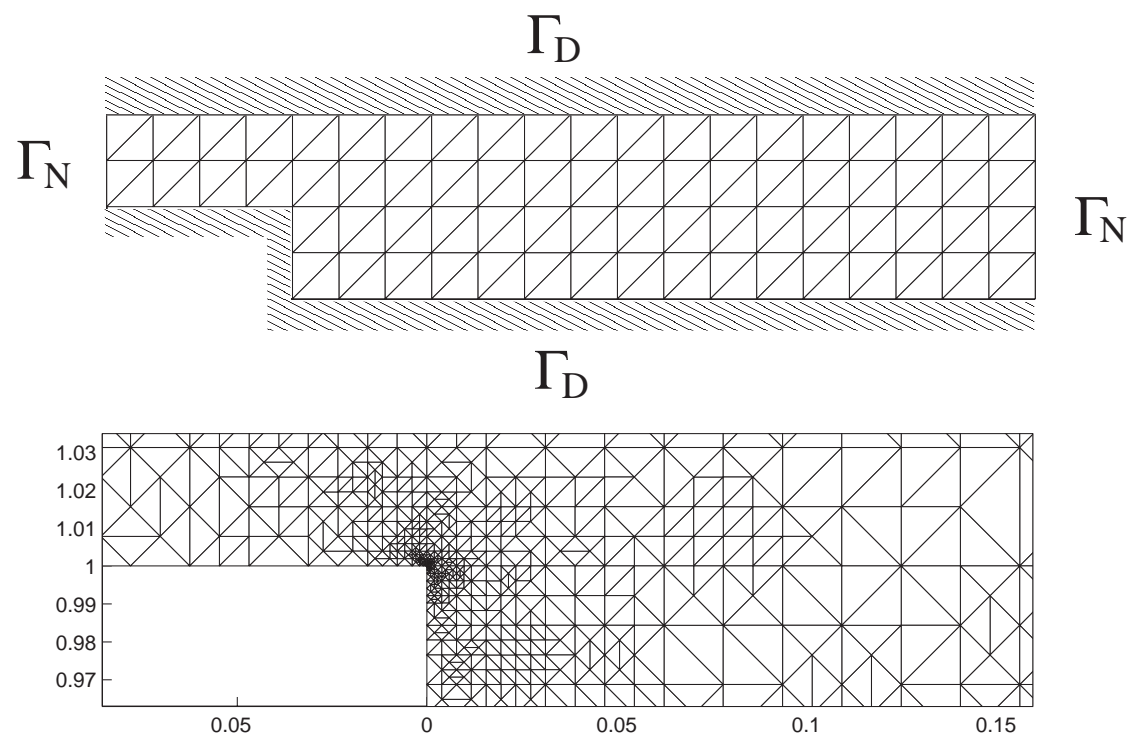

Figure 6 . Mesh $\mathcal{T}_{0}$ and magnified detail at re-entrant corner of Mesh $\mathcal{T}_{9}$ of Example 6.2

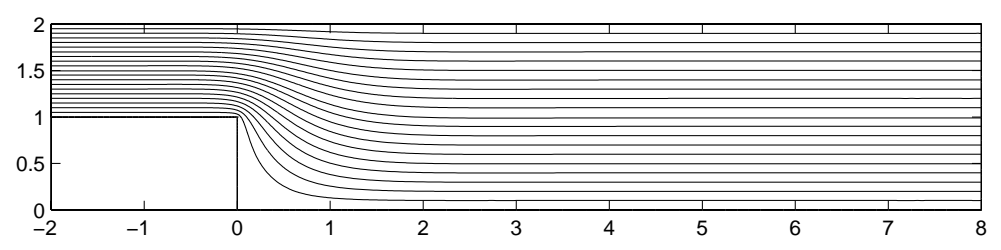

Figure 7. Approximation to streamlines based on $\mathcal{T}_{12}$ of Example 6.2 .

Example 6.2. Finally, we report on a benchmark example. Here, we consider the backward facing step with initial mesh and a magnified detail at re-entrant corner after nine iterations using Algorithm $\left(A_{Z}\right)$ as plotted in Figure $[$ (cf. [BW]). Here, we choose $\mathcal{A}(\alpha)=\alpha / 50$. Neumann boundary conditions are $g:=(68,(2 y-3) / 1100)$ for $x=-2,1 \leq y \leq 2$ and $g:=(17,(1-y) / 4400)$ for $x=8,0 \leq y \leq 2$. On the remaining boundary we define homogeneous Dirichlet conditions.

In Figure 5 we plot the a posteriori error estimates $\eta_{R}$ and $\eta_{Z}$ for uniform and adaptive meshes. The convergence rate of $\eta_{R}$ and $\eta_{Z}$ is approximately 1 for the adaptive meshes and 0.66 for uniform meshes. As expected, the a posteriori estimates $\eta_{R}$ and $\eta_{Z}$ decrease faster for adaptively refined meshes with an optimal convergence rate. If we supposed that $\eta_{z}$ is almost exact, then the error estimator $\eta_{R}$ would overestimate by a factor $\approx 3.1$. The quantities $\eta_{R}$ and $\eta_{Z}$ are smaller on meshes obtained by Algorithm $\left(A_{Z}\right)$ than Algorithm $\left(A_{R}\right)$.

The approximate streamlines based on $\mathcal{T}_{12}$ and Algorithm $\left(A_{Z}\right)$ are plotted in Figure [ in agreement with corresponding pictures in the literature.

In all examples, the meshes are highly nonuniform and the experimental convergence rates of the true and estimated error have been improved to the optimal order which supports the assertion that our adaptive schemes are very useful in practise. 


\section{REFERENCES}

$[\mathrm{ACF}]$ J. Alberty, C. Carstensen, S.A. Funken: Remarks around 50 lines of Matlab: short finite element implementation. Berichtsreihe des Mathematischen Seminars Kiel, Technical report 98-11 Universität Kiel (1998). Num. Alg. 20 (1999) 117-137. http://www.numerik.uni-kiel.de/reports/1998/98-11.html) CMP 2000:01

[A] A. Alonso: Error estimators for a mixed method. Numer. Math. 74 (1996) 385-395. MR 97g:65212

[BB] W. Bao, J.W. Barrett: A priori and a posteriori error bounds for a nonconforming linear finite element approximation of a nonNewtonian flow. RAIRO Modél. Math. Anal. Numér. 32 (1998) 843-858. MR 99i:76086

[BW] R.E. Bank, B.D. Welfert: A posteriori error estimates for the Stokes problem. SIAM Numer. Anal. 28 (1991) 591-623. MR 92a:65284

[BF] F. Brezzi, M. Fortin: Mixed and hybrid finite element methods. Springer-Verlag 1991. MR 92d:65187

[BS] S.C. Brenner, L.R. Scott: The Mathematical Theory of Finite Element Methods. Texts Appl. Math. 15, Springer, New-York, 1994. MR 95f:65001

[Ca1] C. Carstensen: A posteriori error estimate for the mixed finite element method. Math. Comp. 66 (1997) 465-476. MR 98a:65162

[Ca2] : Quasi interpolation and a posteriori error analysis in finite element method. $M^{2} A N$ Math. Model Numer. Anal. 33 (1999) 1187-1202. CMP 2000:07

[CB] C. Carstensen, S. Bartels: Averaging techniques yield reliable error control in low order finite element methods on unstructured grids. Berichtsreihe des Mathematischen Seminars Kiel, Technical report 99-11 Universität Kiel (1999) http://www. numerik.uni-kiel.de/reports/1999/99-11.html

[CD] C. Carstensen, G. Dolzmann: A posteriori error estimates for mixed FEM in Elasticity. Numer. Math. 81 (1998) 187-209. MR 99m:65208

[CJ] C. Carstensen, S. Jansche: A posteriori error estimates for finite element discretization of the Stokes problem. Berichtsreihe des Mathematischen Seminars Kiel, Technical report 97-9 Universität Kiel (1997) (unpublished) http://www . numerik. uni-kiel.de/reports/1997/97-9.html

$[\mathrm{CV}]$ C. Carstensen, R. Verfürth: Edge residuals dominate a posteriori error estimates for low order finite element methods. Berichtsreihe des Mathematischen Seminars Kiel, Technical report 97-6 Universität Kiel (1997) http://www.numerik.uni-kiel.de/reports/1997/97-6.html; SIAM J. Numer. Anal. 36 (1999) 1571-1587. MR 2000g:65115

[Ci] P.G. Ciarlet: The finite element method for elliptic problems. North-Holland, Amsterdam 1978. MR 58:25001]

[Cl] P. Clément: Approximation by finite element functions using local regularization. RAIRO Sér. Rouge Anal. Numér. 9 (1975) no. R-2, 77-84. MR 53:4569

[DDP] E. Dari, R. Duran, and C. Padra: Error estimators for nonconforming finite element approximations of the Stokes problem. Math. Comp. 64 (1995) 1017-1033. MR 95j:65136

[EHJ] K. Eriksson, D. Estep, P. Hansbo, C. Johnson: Introduction to adaptive methods for differential equations. Acta Numerica (1995) 105-158. MR 96k:65057

[FM] R.S. Falk, M. Morley: Equivalence of finite element methods for problems in elasticity. SIAM J. Numer. Anal. 27 (1990) 1486-1505. MR 91i:65177.

[GR] V. Girault, P.A. Raviart: Finite Element Methods for Navier-Stokes Equations. Theory and Algorithms. Springer, Berlin, 1986. Pitman 1985. MR 88b:65129

[Ho] L. Hörmander: Linear Partial Differential Operators. Berlin-Heidelberg-New York: Springer 1963. MR 28:4221

[KS] R. Kouhia, R. Stenberg: A linear nonconforming finite element method for nearly incompressible elasticity and Stokes flow. Comput. Meth. Appl. Mech. Engrg. 124 (1995) 195-212. MR 96d:73073

[LM] J.L Lions, E. Magenes: Nonhomogeneous boundary value problems and applications, Vol. I. Springer, Berlin, 1972. MR 50:2670

[P] C. Padra: A posteriori error estimators for nonconforming approximation of some quasiNewtonian flows. SIAM J. Numer. Anal. 34 (1997) 1600-1615. MR 98h:65050

[QV] A. Quateroni, A. Valli: Numerical Approximation of Partial Differential Equations. Springer, Berlin, 1994. 
[T] R. Temam: Navier-Stokes Equations. North-Holland, Amsterdam, 1985.

[V1] R. Verfürth: A review of a posteriori error estimation and adaptive mesh-refinement techniques. Teubner Skripten zur Numerik. B.G. Willey-Teubner, Stuttgart, 1996.

[V2] R. Verfürth: A posteriori error estimators for the Stokes equations. Numer. Math. 55 (1989) 309-325. MR 90d:65187

[V3] R. Verfürth: A posteriori error estimators for the Stokes equations. II. Nonconforming discetizations. Numer. Math. 60 (1991) 235-249. MR 92j:65189

[ZZ] O.C. Zienkiewicz, J.Z. Zhu: A simple error estimator and adaptive procedure for practical engineering analysis. Int. J. Numer. Meth. Engrg. 24 (1987) 337-357. MR 87m:73055

Mathematisches Seminar, Christian-Albrechts-Universität zU Kiel, Ludewig-MeynStr. 4, D-24098 Kiel, Germany

E-mail address: cc@numerik.uni-kiel.de

Mathematisches Seminar, Christian-Albrechts-Universität Zu Kiel, Ludewig-MeynStr. 4, D-24098 Kiel, Germany

E-mail address: saf@numerik.uni-kiel.de 